\title{
Star formation and stellar populations in the Wolf-Rayet(?) luminous compact blue galaxy IRAS $08339+6517^{\star, \star \star}$
}

\begin{abstract}
Á. R. López-Sánchez, C. Esteban, and J. García-Rojas
Instituto de Astrofísica de Canarias, 38200 La Laguna, Tenerife, Spain

e-mail: angelrls@iac.es

Received 23 March 2005 / Accepted 28 November 2005

\section{ABSTRACT}

Aims. IRAS $08339+6517$ is a luminous infrared and Ly $\alpha$-emitting starburst galaxy that possesses a dwarf companion object at a projected distance of $56 \mathrm{kpc}$. An H I tidal tail has recently been detected between both galaxies, suggesting that about $70 \%$ of the neutral gas has been ejected from them.

Methods. We present deep broad-band optical images, together with narrow band H $\alpha$ CCD images, and optical intermediate-resolution spectroscopy of both galaxies.

Results. The images reveal interaction features between both systems and strong H $\alpha$ emission in the inner part of IRAS 08339+6517. The chemical composition of the ionized gas of the galaxies is rather similar. The analysis of their kinematics also indicates interaction features and reveals an object that could be a candidate tidal dwarf galaxy or a remnant of an earlier merger. Our data suggest that the H I tail has been mainly formed from material stripped from the main galaxy. We find weak spectral features that could be attributed to the presence of Wolf-Rayet stars in this starburst galaxy and estimate an age of the most recent burst of around 4-6 Myr. A more evolved underlying stellar population, with a minimal age between 100-200 Myr, is also detected and fits an exponential intensity profile. A model which combines $85 \%$ young and $15 \%$ old populations can explain both the spectral energy distribution and the H I Balmer and He I absorption lines presented in our spectrum. The star formation rate of the galaxy is consistently derived using several calibrations, giving a value of $\sim 9.5 M_{\odot} \mathrm{yr}^{-1}$.

Conclusions. IRAS 08339+6517 does satisfy the criteria of a luminous compact blue galaxy, rare objects in the local universe but common at high redshifts, being a very interesting target for detailed studies of galaxy evolution and formation.
\end{abstract}

Key words. galaxies: starburst - galaxies: interactions - galaxies: abundances - galaxies: kinematics and dynamics - stars: Wolf-Rayet

\section{Introduction}

Determining the star formation history in galaxies is fundamental for the understanding of their evolution. In nearby galaxies, such as those belonging to the Local Group, it is performed studying the stellar content via color-magnitude diagrams (CMD) and stars as age tracers (see Grebel 1999, and references there in). However, in distant galaxies it is only interpreted by means of spectral synthesis techniques. The analysis of the interstellar medium (ISM) in and around galaxies complements the study of their star formation histories. Star formation activity is stronger in starburst galaxies, where the dominant and very young stellar population could even hide the more evolved stellar population presented in it. The more extreme cases are the blue compact dwarf (BCDs) galaxies, that

* Based on observations made with NOT (Nordic Optical Telescope) operated on the island of La Palma by Instituto de Astrofísica de Canarias in the Spanish Observatories of Roque de Los Muchachos of the Instituto de Astrofísica de Canarias.

$\star \star$ Table 4 is only available in electronic form at http://www. edpsciences.org have very low metal abundances and exhibit a global starburst activity. Although the majority of these systems possesses an old underlying population with ages of several Gyr (Loose \& Thuan 1986), a few of them do not present it, suggesting that they are really young galaxies (Izotov \& Thuan 1999). The best example of this kind of BCDs is I Zw 18, a bona fide young galaxy in the local universe (Izotov \& Thuan 2004). At intermediate and high redshifts a heterogeneous class of vigorous starburst systems with luminosities around $L^{*}\left(L^{\star}=1.0 \times 10^{10} L_{\odot}\right)$ are observed (Guzmán et al. 2003). They are designated as luminous compact blue galaxies (LCBGs) and their evolution and nature are still open questions, being fundamental to get a sample of them in the local universe to investigate the origin of their activity (Werk et al. 2004).

An important subset of starbursts are the so-called Wolf-Rayet (WR) galaxies, whose integrated spectra have a broad emission feature around $4650 \AA$ that has been attributed to WR stars. This feature consists of a blend of N III $\lambda 4640$, C III/C IV $\lambda 4650$ and He II $\lambda 4686$ emission lines (Conti 1991), the last one being the most prominent line. The WR feature indicates the presence of a substantial population of these kinds 
of massive stars whose ages are less than 6 Myr and offers the opportunity to study very young starbursts (Schaerer \& Vacca 1998). Furthermore, they constitute the best direct measure of the upper end of the initial mass function (IMF), a fundamental ingredient for studying unresolved stellar populations (Schaerer et al. 2000; Pindao et al. 2002). Studying a sample of WR galaxies, Méndez \& Esteban (2000) suggested that interactions with or between dwarf objects could be the main star formation triggering mechanism in dwarf galaxies and noted that the interacting and/or merging nature of WR galaxies can be detected only when deep and high-resolution images and spectra are available. The compilation of WR galaxies performed by Schaerer et al. (1999) lists 139 members, but since then this number has increased (Popescu \& Hopp 2000; Bergvall \& Östlin 2002; Contini et al. 2002; Pindao et al. 2002; Lípari et al. 2003; Tran et al. 2003; Fernandes et al. 2004; Izotov et al. 2004; Pustilnik et al. 2004; Kniazev et al. 2004), these galaxies have even been detected at high $z$ (Villar-Martín et al. 2004). In this paper, we add a new member to the list of probable WR galaxies: the luminous infrared galaxy IRAS 08339+6517, which, furthermore, could be also classified as a luminous compact blue galaxy (LCBG).

IRAS $08339+6517$ is at $80 \mathrm{Mpc}$ (at that distance, $1^{\prime \prime}=$ $388 \mathrm{pc}$, assuming $H_{0}=75 \mathrm{~km} \mathrm{~s}^{-1} \mathrm{Mpc}^{-1}$ ) and was firstly reported in the IRAS Point Source Catalog (1986). Margon et al. (1988) performed a multi-wavelength study and described the object as an exceptionally bright and compact starburst nucleus. Wiklind (1989) found abundant molecular gas in IRAS $08339+6517$ from CO observations, concluding that it is a truly starburst system. González-Delgado et al. (1998) presented a detailed UV analysis, suggesting that $\mathrm{O}$ stars must be present in the ionizing cluster(s) of the galaxy in order to explain the blue wing of the C IV $\lambda 1550$ and Si IV $\lambda 1400$ stellar absorption lines, although these stars cannot be more massive than about $40 M_{\odot}$. Kunth et al. (1998) noted a P Cygni profile in the $\operatorname{Ly} \alpha$ emission, which also showed two components indicating the chaotic structure of the interstellar medium in this object. Mas-Hesse et al. (2003) included IRAS 08339+6517 in their HST UV and optical study of Ly $\alpha$ starbursts.

In a very recent paper, Cannon et al. (2004) presented VLA HI imaging of this starburst galaxy and found an extended tidal structure in neutral hydrogen indicating that it is interacting with a nearby companion, 2MASX J08380769+6508579 (which we designate as the companion galaxy throughout this paper). This feature provides an evidence for tidally induced starburst episodes. Cannon et al. (2004) estimated neutral hydrogen masses of $(1.1 \pm 0.2) \times 10^{9} M_{\odot}$ and $(7.0 \pm 0.9) \times 10^{8} M_{\odot}$ for IRAS 08339+6517 and its companion galaxy, respectively, and a mass of $(3.8 \pm 0.5) \times 10^{9} M_{\odot}$ in the tidal material between them. Consequently, $\sim 70 \%$ of the neutral gas has been removed from one or both galaxies.

We are developing a detailed analysis of Wolf-Rayet galaxies using both deep images and spectra in optical wavelenghts. Our data show increasing evidences of the connection between the detection of WR features and the finding of interaction signatures in this kind of starbursts (Méndez \& Esteban 2000; López-Sánchez \& Esteban 2003; López-Sánchez et al. 2004a,b;
Table 1. Summary of observations performed using ALFOSC in $2.56 \mathrm{~m}$ NOT, with a spatial resolution of $0.19^{\prime \prime} \mathrm{pix}^{-1}$. The position angle, spectral resolution and wavelength range of the intermediate resolution spectroscopy is shown in the last line.

\begin{tabular}{cccc}
\hline \hline Observations & Date & Exp. time (s) & Filter \\
\hline Broad-band & $05 / 04 / 03$ & $3 \times 300$ & $U$ \\
imaging & $05 / 04 / 03$ & $3 \times 300$ & $B$ \\
& $04 / 03 / 20$ & $2 \times 300$ & $V$ \\
& $04 / 03 / 20$ & $3 \times 300$ & $R$ \\
Narrow-band & $04 / 03 / 20$ & $3 \times 300$ & $\mathrm{H} \alpha$ (F.19) \\
imaging & $04 / 03 / 20$ & $2 \times 300$ & $\mathrm{H} \alpha$ cont. (F.20) \\
Intermediate & $04 / 03 / 20$ & $3 \times 900$ & grism \#7 \\
resolution & $\mathrm{PA}$ & $R$ & $\Delta \lambda$ \\
spectroscopy & $138^{\circ}$ & $1.5 \AA$ pix $^{-1}$ & $3600-6800 \AA$ \\
\hline
\end{tabular}

López-Sánchez 2006). The characteristics previously observed in IRAS 08339+6517 (strong $\mathrm{H} \alpha$ emission, starburst nature and the detection of an extended H I tidal tail in the direction of its companion dwarf galaxy) make it an ideal target to look for the existence of WR stars. In this way, we have performed deep broad-band optical and narrow-band H $\alpha$ CCD images together optical intermediate-resolution spectroscopy of IRAS 08339+6517 and its dwarf companion galaxy. Our main aim is to study their morphology and stellar populations, as well as the distribution, chemical composition and kinematics of the ionized gas, and to check if WR stars are presented in their youngest bursts.

\section{Observations and data reduction}

The main data were obtained on 2004 March 20 at the $2.56 \mathrm{~m}$ Nordic Optical Telescope (NOT) at Roque de los Muchachos Observatory (La Palma, Canary Islands, Spain). We used the ALFOSC (Andalucía Faint Object Spectrograph and Camera) instrument in both image and spectrograph mode, with a Loral/Lesser CCD detector $(2048 \times 2048$ pixels $)$ with a pixel size of $13.5 \mu \mathrm{m}$ and spatial resolution of $0.19^{\prime \prime}$ pixel $^{-1}$. Additional $U$ and $B$ images were obtained on 2005 April 03 using the same telescope and instrument. The journal of all the imaging and spectroscopical observations can be found in Table 1 .

\subsection{Optical imaging}

We obtained three 300 second exposures for $U, B$ and $R$ standard Johnson filters and two 300 second exposures for the $V$ filter that were combined to obtain the final images with a PSF FWHM of $0.8^{\prime \prime}$. The standard starfield SA107-602 (Landolt 1992) was used to flux calibrate the final images. It was observed at different airmasses during the night. Twilight images of different zones of the sky (blank fields) were taken for each filter in order to perform the flat-field correction. All the reduction process (bias subtraction, flat-fielding and flux calibration) 
was done with $\mathrm{IRAF}^{1}$ package following standard procedures. IRAF software was also used to determine the $3 \sigma$ contours over the sky background level and to obtain the photometric values of the galaxies.

\subsection{Ho imaging}

The narrow-band filters for $\mathrm{H} \alpha$ and red-continuum images (centered at 6687 and $6571 \AA$ and with a $F W H M$ of 50 and $47 \AA$, respectively) were selected taking into account the recession velocity of the object given by the NASA/IPAC Extragalactic Database (NED). Three (two for the continuum) exposures of 300 seconds were added to obtain the final image in each filter. The reduction process was similar to the broadband images. The absolute flux calibration was achieved by taking short exposures of the spectrophotometric standard star Feige 56 (Hamuy et al. 1992) at different airmasses.

To obtain the continuum-subtracted $\mathrm{H} \alpha$ image, we followed the standard procedure given by Cedrés (2003). A scaling factor between the $\mathrm{H} \alpha$ and the continuum frames was determined using non-saturated field stars (in our case, this scaling factor was 0.9 ). The $F W H M$ of the PSF for both images was similar (around $0.6^{\prime \prime}$ ), so no Gaussian filter was applied. The finally flux-calibrated and continuum-subtracted $\mathrm{H} \alpha$ image was then produced by subtracting the scaled continuum frame from the $\mathrm{H} \alpha$ frame. The $\mathrm{W}(\mathrm{H} \alpha)$ image was also obtained following the standard procedure, correcting the $\mathrm{W}(\mathrm{H} \alpha)$ for the underlying stellar population of the disk.

\subsection{Intermediate-resolution spectroscopy}

We used ALFOSC in spectrographic mode with a slit of $6.4^{\prime}$ (the field of view of the camera) long and $1^{\prime \prime}$ wide to obtain intermediate-resolution spectroscopy of the galaxies. Grism \#7 with 600 rules $\mathrm{mm}^{-1}$ and resolution of 1300 was used, with a dispersion of $111 \AA \mathrm{mm}^{-1}$ and spectral resolution of $1.5 \AA \mathrm{pix}^{-1}$. Each spectrum spans the wavelength range 3200 to $6800 \AA$ (redshift corrected). The slit position was located at $\mathrm{PA}=138^{\circ}$, which was chosen in order to cross the center of IRAS $08339+6517$ and its companion galaxy. Three $15 \mathrm{~min}$ exposures were taken and combined to obtain good signal-tonoise and an appropriate removal of cosmic rays in the final spectra. We used comparison He and Ne lamp exposures for the wavelength calibration of the spectra. The absolute flux calibration was achieved by observations of the standard star Feige 56 (Massey et al. 1988). The observations were made at air masses between 1.1 and 1.3. Consequently, no correction was made for atmospheric differential refraction.

All the CCD frames for spectroscopy were reduced using standard IRAF procedures to perform bias correction, flatfielding, cosmic-ray rejection, wavelength and flux calibration, and sky subtraction. The correction for atmospheric extinction was performed using an average curve for the continuous atmospheric extinction at Roque de los Muchachos Observatory. For the two-dimensional spectra two different apertures were

1 IRAF is distributed by NOAO which is operated by AURA Inc., under cooperative agreement with NSF. defined along the spatial direction to extract the final onedimensional spectra of the galaxies, centering each aperture on its brightest zone. Small two-dimensional distortions were corrected by fitting the maxima of [O II] $\lambda \lambda 3726,3729$ doublet and $\mathrm{H} \alpha$ emission lines (between 2 and 3 pixels, $0.4-0.6^{\prime \prime}$ ).

\section{Results}

\subsection{The extinction toward IRAS $08339+6517$}

We have carefully studied the reddening coefficient, $C(\mathrm{H} \beta)$, derived from our spectra using the Balmer decrement (see Sect. 3.4) in order to achieve accurate photometric and spectroscopic data of IRAS $08339+6517$ and its companion galaxy. However, the fluxes of nebular Balmer lines in the optical spectra must be also corrected for underlying stellar absorption. In this way, we performed an iterative procedure to derive the reddening coefficient and the equivalent widths of the absorption in the hydrogen lines, $W_{\text {abs }}$, to correct the observed line intensities for both effects. We assumed that the equivalent width of the absorption components is the same for all the Balmer lines and used the relation given by Mazzarrella \& Boronson (1993) to the absorption correction,

$C(\mathrm{H} \beta)=\frac{1}{f(\lambda)} \log \left[\frac{\frac{I(\lambda)}{I(\mathrm{H} \beta)} \times\left(1+\frac{W_{\mathrm{abs}}}{W_{\mathrm{H} \beta}}\right)}{\frac{F(\lambda)}{F(\mathrm{H} \beta)} \times\left(1+\frac{W_{\mathrm{abs}}}{W_{\lambda}}\right)}\right]$,

for each detected hydrogen Balmer line. In this equation, $F(\lambda)$ and $I(\lambda)$ are the observed and the theoretical fluxes (unaffected by reddening or absorption); $W_{\mathrm{abs}}, W_{\lambda}$ and $W_{\mathrm{H} \beta}$ are the equivalent widths of the underlying stellar absorption, the considered Balmer line and $\mathrm{H} \beta$, respectively, and $f(\lambda)$ is the reddening curve normalized to $\mathrm{H} \beta$ using the Seaton (1979) law. We have used the pairs $\mathrm{H} \alpha / \mathrm{H} \beta, \mathrm{H} \gamma / \mathrm{H} \beta$ and $\mathrm{H} \delta / \mathrm{H} \beta$ for the main galaxy but only the two first line ratios for the companion object. We have considered the theoretical ratios between these pairs of H I Balmer lines expected for case B recombination given by Storey \& Hummer (1995) and appropriate electron temperatures around $10^{4} \mathrm{~K}$ and electron densities around $100 \mathrm{~cm}^{-3}(\mathrm{H} \alpha / \mathrm{H} \beta=2.86, \mathrm{H} \gamma / \mathrm{H} \beta=0.469$ and $\mathrm{H} \delta / \mathrm{H} \beta=0.259)$. The $C(\mathrm{H} \beta)$ and $W_{\text {abs }}$ that provide the best match between the corrected and the theoretical line ratios are shown in Table 4.

Once we have obtained the reddening coefficient, the photometric data can also be corrected for extinction by interstellar dust. We used the standard Seaton (1979) law and the standard ratio of $R_{V}=A_{V} / E(B-V)=3.1$ to obtain the extinction in $V, A_{V} \sim 2.11 \times C(\mathrm{H} \beta)$ and the color excess, $E(B-V)=0.68 \times C(\mathrm{H} \beta)$. The data in $U, B$ and $R$ were corrected using $A_{U}=1.531 \times A_{V}, A_{B}=1.324 \times A_{V}$ and $A_{R}=0.748 \times A_{V}$ (Rieke \& Lebofsky 1985), whereas the data in $\mathrm{H} \alpha$ filter were corrected using $A_{\mathrm{H} \alpha}=1.61 \times C(\mathrm{H} \beta)$ (Seaton 1979).

As we explain in Sect. 3.4, we have extracted eight different apertures to study the ionized gas in IRAS $08339+6517$. The $C(\mathrm{H} \beta)$ derived varies between 0.18 and 0.30 , implying a color excess between 0.12 and 0.20 . We have adopted the average $C(\mathrm{H} \beta)(=0.24)$ of these eight zones as representative for the galaxy. The derived color excess used to correct the photometric data is $E(B-V)=0.16$ (see Table 2). 
Table 2. General properties of the galaxies.

\begin{tabular}{|c|c|c|}
\hline Property & IRAS $08339+6517$ & companion \\
\hline$M_{B}^{0}$ & $-21.57 \pm 0.04$ & $-18.21 \pm 0.04$ \\
\hline$m_{B}^{0}$ & $12.94 \pm 0.04$ & $16.31 \pm 0.04$ \\
\hline$L_{B} / L_{\odot}$ & $(6.69 \pm 0.24) \times 10^{10}$ & $(2.99 \pm 0.11) \times 10^{9}$ \\
\hline$E(B-V)^{a}$ & $0.16 \pm 0.02$ & $0.13 \pm 0.02$ \\
\hline$(U-B)^{0}$ & $-0.51 \pm 0.08$ & $-0.16 \pm 0.10$ \\
\hline$(B-V)^{0}$ & $0.02 \pm 0.08$ & $0.20 \pm 0.08$ \\
\hline$(V-R)^{0}$ & $0.24 \pm 0.08$ & $0.26 \pm 0.08$ \\
\hline$(V-J)^{0, \mathrm{~b}}$ & $1.39 \pm 0.06$ & $1.56 \pm 0.12$ \\
\hline$(J-H)^{0, \mathrm{~b}}$ & $0.64 \pm 0.05$ & $0.21 \pm 0.25$ \\
\hline$\left(H-K_{\mathrm{s}}\right)^{0, \mathrm{~b}}$ & $0.23 \pm 0.06$ & $0.68 \pm 0.28$ \\
\hline$M_{\mathrm{H} \text { II }}\left(M_{\odot}\right)$ & $(1.45 \pm 0.07) \times 10^{7}$ & $(2.6 \pm 0.3) \times 10^{5}$ \\
\hline$M_{\mathrm{HI}}\left(M_{\odot}\right)^{c}$ & $(1.1 \pm 0.2) \times 10^{9}$ & $(7.0 \pm 0.9) \times 10^{8}$ \\
\hline$M_{\mathrm{H}_{2}}\left(M_{\odot}\right)^{d}$ & $\sim 1.2 \times 10^{9}$ & $\ldots$ \\
\hline$M_{\text {dust }}\left(M_{\odot}\right)^{e}$ & $4.5 \times 10^{6}$ & $\ldots$ \\
\hline$M_{\text {Kep }}\left(M_{\odot}\right)$ & $(10 \pm 3) \times 10^{9}$ & $(8 \pm 2) \times 10^{9}$ \\
\hline$M_{\mathrm{Kep}} / M_{\mathrm{HI}}$ & 9.1 & 1.1 \\
\hline$M_{\mathrm{H} \text { II }} / M_{\mathrm{HI}}$ & 0.013 & 0.0004 \\
\hline$L_{B} / M_{\mathrm{Kep}}$ & 6.7 & 0.37 \\
\hline$L_{B} / M_{\mathrm{HI}}$ & 60.8 & 4.2 \\
\hline$L_{B} / M_{\text {dust }}$ & $1.5 \times 10^{4}$ & $\cdots$ \\
\hline $\operatorname{SFR}\left(M_{\odot} \mathrm{yr}^{-1}\right)$ & 9.5 & 0.17 \\
\hline$\tau_{\text {gas }}(\mathrm{Gyr})$ & 0.15 & 5.4 \\
\hline $\operatorname{Age}^{f}(\mathrm{Myr})$ & $4-5$ & $5-6$ \\
\hline $\operatorname{Age}^{g}(\mathrm{Myr})$ & $30-50$ & $>250$ \\
\hline$\Delta v_{r}^{h}\left(\mathrm{~km} \mathrm{~s}^{-1}\right)$ & 0 & $20 \pm 10$ \\
\hline $12+\log \mathrm{O} / \mathrm{H}^{\mathrm{i}}$ & $8.45 \pm 0.10$ & $8.38 \pm 0.10$ \\
\hline$N(\mathrm{WR})^{j}$ & $\sim 310$ & $\ldots$ \\
\hline $\mathrm{WR} /(\mathrm{WR}+\mathrm{O})^{j}$ & 0.03 & $\ldots$ \\
\hline
\end{tabular}

${ }^{a}$ Derived from $C(\mathrm{H} \beta)$. See Sect. 3.1 for more details.

${ }^{b}$ Derived from the values of 2MASS shown by the NED.

${ }^{c}$ From Cannon et al. (2004).

${ }^{d}$ From Wiklind (1989).

${ }^{e}$ Derived using the Huchtmeier et al. (1995) relation between the 60 and $100 \mu \mathrm{m}$ FIR fluxes and the dust mass (last equation in p. 678).

${ }^{f}$ Estimated age of the most recent bursts using our $\mathrm{H} \alpha$ and spectroscopical data. See Sect. 4.1 for more details.

${ }^{g}$ Estimated age comparing our optical colors with the STARBURSTS 99 (Leitherer et al. 1999) and PEGASE.2 (Fioc \& Rocca-Volmerange 1997) models. See Sect. 4.1 for more details.

${ }^{h}$ Radial velocity with respect to the center of IRAS $08339+6517$.

${ }^{i}$ Derived using the Pilyugin (2001) empirical calibration (see Sect. 3.4.2).

${ }^{j}$ Approximate number of $\mathrm{WR}$ stars and $\mathrm{WR} /(\mathrm{WR}+\mathrm{O})$ ratio (see Sect. 4.3).

Schlegel et al. (1998) presented an all-sky map of infrared dust emission, determining a color excess $E(B-V)=0.092$ (assuming $R_{V}=3.1$ ) at the Galactic longitude and latitude of IRAS $08339+6517$. This value is lower than the $E(B-V)$ we obtain for the galaxies $(0.16$ and 0.13$)$, indicating that a considerable fraction of the dust in the line of sight is external to the Milky Way, especially in the main object.
The importance of the extinction correction was previously remarked on by González-Delgado et al. (1998), who studied the variation of the color excess derived from various methods: the Balmer decrement (that gave an $E(B-V)=0.52$, a similar value that the one derived by Margon et al. (1988) using the $\mathrm{H} \alpha / \mathrm{H} \beta$ ratio, $E(B-V)=0.55$, although they do not correct for the underlying stellar absorption) and the change of the UV continuum slope (which indicates lower values for the extinction, $E(B-V)=0.1-0.2)$. They explain this difference in terms of a nonhomogeneous distribution of the dust, although it could also be due to the variation of the evolutionary stage of the stellar population with the aperture size. The data derived from our optical spectra suggest that $E(B-V)$ is not so high as those derived by González-Delgado et al. (1998) and Margon et al. (1988) using the same method but is similar to the value derived from the UV data. This result is supported by Stevens \& Strickland (1998), who studied ROSAT PSPC seredipitious observations of IRAS $08339+6517$ and concluded that the spectral fitting yields an X-ray temperature of $k T=0.58 \mathrm{keV}$, with $Z=0.02 Z_{\odot}$ and a column of $1.40 \times 10^{21} \mathrm{~cm}^{-2}$, implying substantial local absorption. Using the dust-to-gas ratio given by Kent et al. (1991), a color excess of 0.24 is derived, a bit higher than the one we have assumed from the Balmer decrement, but not as high as that derived by González-Delgado et al. (1998).

However, we have noted significant differences in $C(\mathrm{H} \beta)$ derived for different zones and apertures inside IRAS 08339+6517. We briefly discuss this in Sect. 4.4.

\subsection{Optical imaging}

In Fig. 1a we show our final deep $R$ image, which has been saturated to show the faintest structures. This deep image reveals a disturbed morphology in the outer regions of IRAS $08339+6517$. The most relevant are a bright ray at the south of the galaxy and a long arc of material connecting the north of the galaxy with the southern bright ray. This arc seems to be broken into two parts to the SE of the galaxy. Another weaker arc goes from the west towards the end of the bright ray. Furthermore, a very diffuse plume is found in the NW of the galaxy, precisely in the direction towards the companion galaxy. This plume coincides with the beginning of the tidal H I material discovered by Cannon et al. (2004) between the two galaxies.

The non-saturated images of the galaxies are shown in Figs. $1 \mathrm{~b}$ and c. IRAS 08339+6517 shows a circular morphology. Assuming that it has a disk geometry, this fact would indicate that the galaxy is nearly face on. Several structures are found in its central areas following an apparently symetrical pattern from the nucleus. Although they are not clearly resolved, they show lower $U-B$ values and possess important $\mathrm{H} \alpha$ emission features (see next section), indicating that the inner area of the galaxy has relatively high star-formation activity. These facts could suggest the presence of a disk-like structure inside IRAS 08339+6517.

The companion galaxy has a not very excentric elliptical morphology, with no relevant feature inside it. It shows bluer colors in its external areas than in its center. 

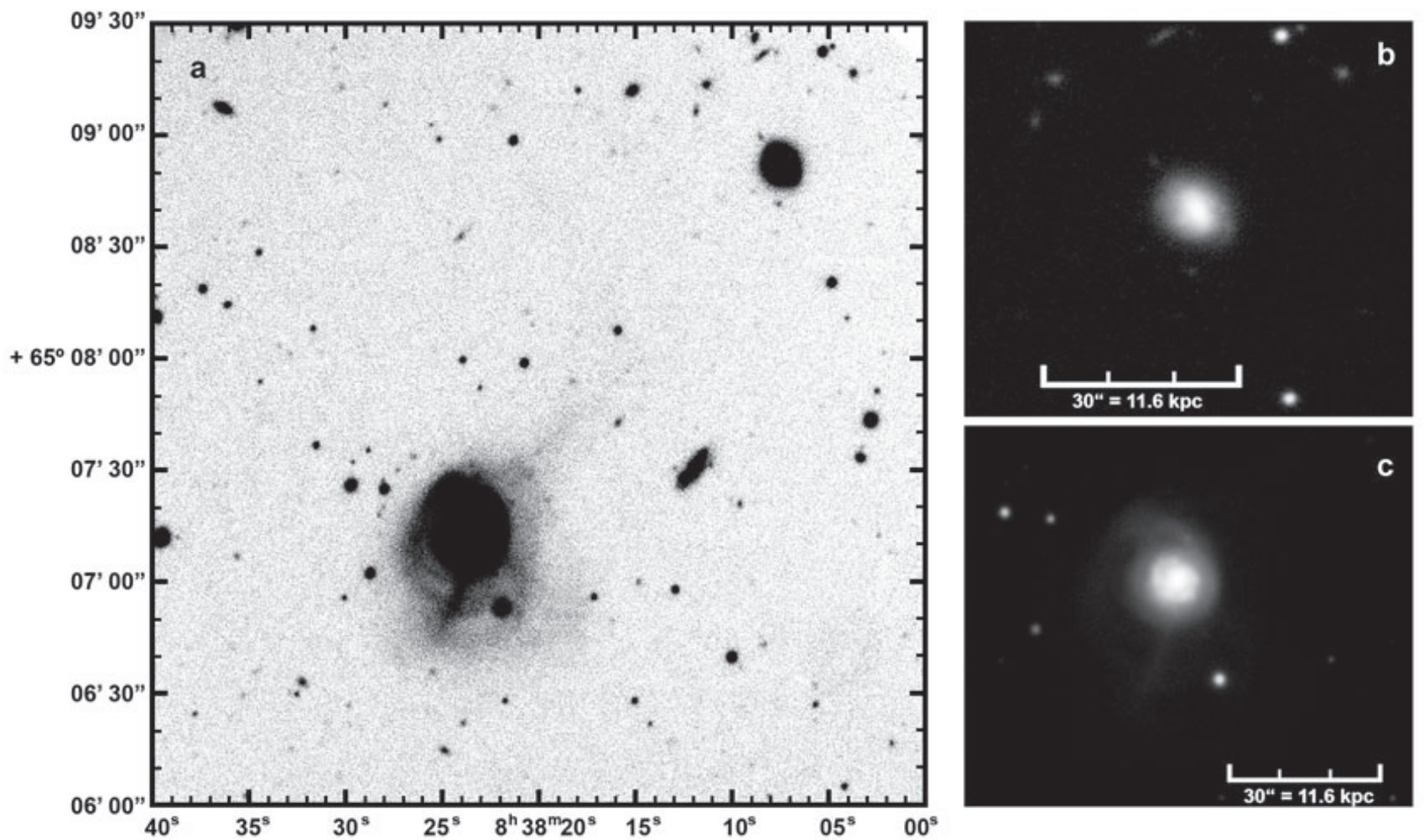

Fig. 1. a) Deep optical image of IRAS $08339+6517$ and its companion galaxy, 2MASX J08380769+6508579, in $R$. It has been saturated to reveal the weakest features. b) The companion galaxy and c) IRAS $08339+6517$ in non-satured $R$ images. The grayscale is in logarithmic scale in all three cases.

We have performed integrated aperture photometry for the galaxies in the optical filters. We have determined the area for which we have integrated the flux using the $3 \sigma$ level isophote over the average sky level in the $B$ image. We have used the same area in $U, V$ and $R$, correcting all values for reddening as indicated in the previous section. We have determined the errors for the photometry by considering the FWHM of the PSF, sky level and flux calibration for each frame.

The final integrated photometric values derived for each galaxy are listed in Table 2. We also show the NIR colors derived from the values given by 2 MASS, that are also corrected by reddening. IRAS $08339+6517$ has bluer colors than its companion object. A detailed analysis of the surface brightness reveals the presence of two different stellar populations inside IRAS 08339+6517, as we will see in Sect. 4.1. The $B$ magnitudes derived implying an absolute blue magnitude of $M_{B} \sim-21.6$ for the main galaxy and $M_{B} \sim-18.2$ for its companion galaxy.

\section{3. $\mathrm{H} \alpha$ imaging}

The $\mathrm{H} \alpha$ map of IRAS $08339+6517$ is shown in Fig. 2. The slit position used for spectroscopy, as well as different regions inside the galaxy that we have also extracted and analyzed, are indicated and labeled. The galaxy has an almost circular morphology in $\mathrm{H} \alpha$ emission, except for the arm located to its northwest (region \#7 in Fig. 2). This feature is also in the direction of the companion galaxy. Several star-formation knots are

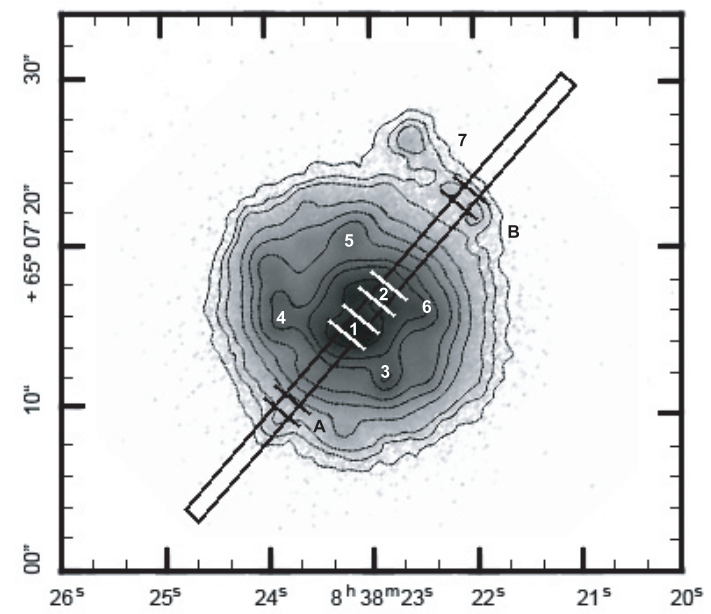

Fig. 2. Continuum-subtracted $\mathrm{H} \alpha$ image of IRAS $08339+6517$. The contours represent $3 \sigma, 5 \sigma, 7 \sigma, 15 \sigma, 30 \sigma, 70 \sigma, 140 \sigma, 230 \sigma, 430 \sigma$ and $700 \sigma$ above the sky level. We also show the position of the slit used for spectroscopy, with PA $138^{\circ}$, and label the different individual regions that we have analyzed.

found inside the disk, the central (region \#1) being the brightest one. This indicates that the entire galaxy is undergoing a considerable star-formation activity. Regions \#4 (which seems to be connected to \#1 and is broken into three small knots) and \#6 (connected to \#2) are elliptic and extend from the center to the outer regions, suggesting diffuse spiral arms. The NW arm also 
Table 3. $\mathrm{H} \alpha$ properties of the galaxies and studied knots inside IRAS $08339+6517$.

\begin{tabular}{|c|c|c|c|c|c|c|}
\hline Knot & Flux $\left(10^{-13} \mathrm{erg} \mathrm{cm}^{-2} \mathrm{~s}^{-1}\right)$ & Luminosity $\left(10^{41} \mathrm{erg} \mathrm{s}^{-1}\right)$ & $\operatorname{SFR}\left(M_{\odot} \mathrm{yr}^{-1}\right)$ & $M_{\mathrm{HII}}\left(10^{6} M_{\odot}\right)$ & $-W(\mathrm{H} \alpha)(\AA)$ & Age (Myr) \\
\hline IRAS 08339+6517 & $16.0 \pm 0.7$ & $12.0 \pm 0.6$ & $9.5 \pm 0.5$ & $14.5 \pm 0.7$ & $110 \pm 10$ & $4.4 \pm 0.2$ \\
\hline \# 1 & $4.3 \pm 0.3$ & $3.3 \pm 0.2$ & $2.62 \pm 0.18$ & $4.1 \pm 0.3$ & $150 \pm 15$ & $3.3 \pm 0.2$ \\
\hline \# 2 & $2.3 \pm 0.2$ & $1.74 \pm 0.14$ & $1.38 \pm 0.11$ & $2.09 \pm 0.17$ & $103 \pm 9$ & $4.4 \pm 0.2$ \\
\hline \#3 & $0.47 \pm 0.05$ & $0.36 \pm 0.04$ & $0.28 \pm 0.03$ & $0.43 \pm 0.04$ & $129 \pm 13$ & $3.9 \pm 0.2$ \\
\hline$\# 4$ & $0.76 \pm 0.08$ & $0.57 \pm 0.06$ & $0.45 \pm 0.05$ & $0.69 \pm 0.07$ & $101 \pm 10$ & $4.4 \pm 0.2$ \\
\hline \# 5 & $0.24 \pm 0.03$ & $0.18 \pm 0.02$ & $0.14 \pm 0.02$ & $0.22 \pm 0.03$ & $95 \pm 11$ & $4.5 \pm 0.3$ \\
\hline \# 6 & $0.70 \pm 0.07$ & $0.53 \pm 0.05$ & $0.42 \pm 0.04$ & $0.63 \pm 0.06$ & $102 \pm 13$ & $4.4 \pm 0.3$ \\
\hline \# 7 (NW arm) & $0.096 \pm 0.015$ & $0.073 \pm 0.011$ & $0.06 \pm 0.01$ & $0.087 \pm 0.013$ & $40 \pm 6$ & $5.4 \pm 0.3$ \\
\hline Companion & $0.28 \pm 0.03$ & $0.21 \pm 0.02$ & $0.17 \pm 0.02$ & $0.26 \pm 0.03$ & $31 \pm 5$ & $5.5 \pm 0.3$ \\
\hline
\end{tabular}

shows three knots of weak $\mathrm{H} \alpha$ emission. Only the brightest is found in the optical images, showing $U-B \sim-0.4, B-V \sim 0.1$ and $V-R \sim 0.2$ colors. The companion galaxy is weaker in $\mathrm{H} \alpha$ emission but it also shows star-formation activity. Its morphology is irregular and split into several weak knots located not in its center but in its outer areas.

We obtained the $\mathrm{H} \alpha$ fluxes for both galaxies and some regions inside IRAS $08339+6517$ using this flux-calibrated continuum-subtracted $\mathrm{H} \alpha$ image. The boundaries of the zones were determined selecting each distinct emission peak as a separate region by eye. We have corrected the $\mathrm{H} \alpha$ fluxes for both reddening (see Sect. 3.1) and [N II] contamination. The [N II] contamination was corrected using the [N II] $\lambda 6583 / \mathrm{H} \alpha$ and [N $\mathrm{NI}] \lambda 6548 / \mathrm{H} \alpha$ ratios derived from our spectra and the transmittance value of the $\mathrm{H} \alpha$ filter for these wavelengths. In the zones for which we have no spectra we used the average $[\mathrm{N} \mathrm{II}] / \mathrm{H} \alpha$ ratios obtained. The errors for the $\mathrm{H} \alpha$ flux was determined considering the $F W H M$ of the PSF, sky level, the flux calibration, and the contributions of the reddening and [N II] contamination, which vary between $4 \%$ (for the main galaxy) and $16 \%$ (for the NW arm). The final fluxes are shown in Table 3. The $\mathrm{H} \alpha$ flux derived for IRAS $08339+6517$ is in excellent agreement with that obtained by Margon et al. (1988), $F_{\mathrm{H} \alpha}=1.3 \times 10^{-12} \mathrm{erg} \mathrm{s}^{-1} \mathrm{~cm}^{-2}$.

The luminosity of the galaxies and regions was calculated assuming a distance of $80 \mathrm{Mpc}$ for the system. For comparison, the giant extragalactic $\mathrm{H}$ II region 30 Doradus in the LMC has an $\mathrm{H} \alpha$ luminosity of $1.5 \times 10^{40} \mathrm{erg} \mathrm{s}^{-1}$ (Kennicutt 1984), similar to region \#5. Assuming that the ionization is due to hot stars and that a single $\mathrm{O} 7 \mathrm{~V}$ star contributes with a luminosity of $L_{\mathrm{H} \alpha}=1.36 \times 10^{36} \mathrm{erg} \mathrm{s}^{-1}$ (Schaerer \& Vacca 1998) to the total flux, we estimated a population of around $\sim 880000$ O7V stars for IRAS $08339+6517$.

We have derived the star formation rate $(S F R)$ and the mass of the ionized gas, $M_{\mathrm{HII}}$, using the standard Kennicutt (1998) calibration. For IRAS $08339+6517$ we find a $S F R_{\mathrm{H} \alpha}=$ $9.5 M_{\odot} \mathrm{yr}^{-1}$, which is in excellent agreement with previous studies. We will compare the SFR derived from different wavelengths in Sect. 4.2. For the mass of the ionized gas, we have derived a value of $M_{\mathrm{HII}} \sim 1.5 \times 10^{7} M_{\odot}$.

We have obtained the equivalent width of the $\mathrm{H} \alpha$ emission, $W(\mathrm{H} \alpha)$, of the galaxies and the knots inside IRAS $08339+6517$ from the $\mathrm{H} \alpha$ image. The results are compiled in Table 3. All the objects present very similar $W(\mathrm{H} \alpha)$, except the center of the galaxy (knot\#1, which has the highest value) and the companion galaxy and the NW arm (both showing lowest values). The equivalent width of the $\mathrm{H} \alpha$ emission can be used to estimate the age of the bursts. We have performed this estimation using the STARBURST99 (Leitherer et al. 1999) models for an instantaneous starburst (see Sect. 4.1 for details). The results for each object are found in Table 3.

Studying the complex structure of the Ly $\alpha$ emission observed in IRAS 08339+6517, Mas-Hesse et al. (2003) conclude that an expanding superbubble of ionized gas powered by the starburst is moving toward the observer at $300 \mathrm{~km} \mathrm{~s}^{-1}$ with respect to the central H II region. This is supported by the detection of X-ray emission with Einstein (Gioia et al. 1990) and ROSAT (Stevens \& Strickland 1998) observatories. The strong $\mathrm{H} \alpha$ emission detected throughout the disk of the galaxy is compatible with this scenario.

\subsection{Intermediate resolution spectra}

The slit position was placed crossing the center of both galaxies, with a position angle of $138^{\circ}$. In Fig. 2 we show the slit position over the $\mathrm{H} \alpha$ map of IRAS $08339+6517$; the $\mathrm{H} \alpha$ flux distribution along the slit is shown in Fig. 3. We have also marked in this figure the apertures used to extract the individual 1-D spectra. The two brightest peaks correspond with the central object (knot \#1) and knot \#2 that we previously detected in the $\mathrm{H} \alpha$ image. We have also extracted two external zones, $\mathrm{A}$ and $\mathrm{B}$, where weak peaks of $\mathrm{H} \alpha$ emission are detected. All four apertures have a size of $1^{\prime \prime} \times 1^{\prime \prime}$. But we have also extracted four additional spectra with increasing apertures to analyze the dependence of the properties of the ionized gas with the aperture size. These new apertures have a size of $2.5^{\prime \prime}, 5^{\prime \prime}, 9^{\prime \prime}$, and $12^{\prime \prime} \times 1^{\prime \prime}$, and are also indicated in Fig. 3 . The best $\mathrm{S} / \mathrm{N}$ ratio is found in the largest aperture, corresponding to the integrated spectrum of IRAS $08339+6517$. This aperture collects approximately $96 \%$ of the total flux of the galaxy observed by our slit. Only one spectrum of the companion galaxy was extracted. Its aperture size was $6.5^{\prime \prime} \times 1^{\prime \prime}$.

The wavelength- and flux-calibrated spectra of the $12^{\prime \prime} \times 1$ " aperture for IRAS $08339+6517$ and the single aperture for the companion galaxy are shown in Fig. 4. The spectrum of IRAS $08339+6517$ shows a high continuum level and an 


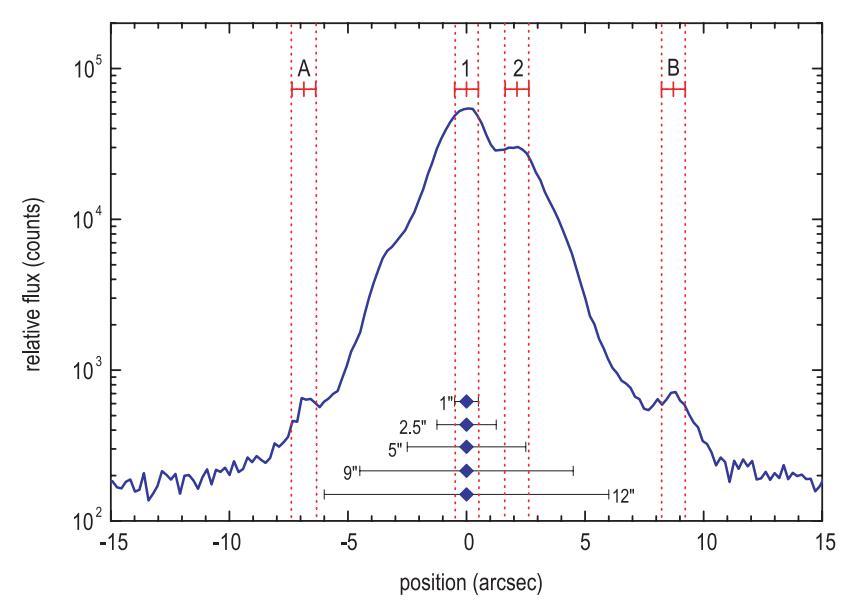

Fig. 3. Relative $\mathrm{H} \alpha$ flux along the slit (see Fig. 2). The different zones for which we have extracted spectra are indicated and labeled. Knots \#1 (the center of the galaxy), \#2, A and B are $1^{\prime \prime}$ in size. We also show four additional apertures extracted to study spatial variations of the ionized gas inside the galaxy. They have $2.5^{\prime \prime}, 5^{\prime \prime}, 9^{\prime \prime}$ and $12^{\prime \prime}$ in size. The aperture of $12^{\prime \prime}$ represents the integrated spectrum of the galaxy, that we show in Fig. 4 (top).

intense underlying stellar absorption in H I Balmer lines. All of them, except $\mathrm{H} \alpha$, show intense absorption wings. This was previously noted by Mas-Hesse et al. (2003). Furthermore, we have detected the main absorption features present in this spectral range: Ca II H $\lambda 3933$, G-band $\lambda 4304, \mathrm{Mg}$ I $\lambda \lambda 5167,87$ and $\mathrm{Na}$ D1 $\lambda 5889$, as well as some He I absorption. These features, together the high continuum level of the spectrum, can be interpreted as the product of a substantial population of older stars, with ages much greater than 10 Myr. Consequently, at least two separate stellar populations are present in the galaxy (see Sect. 4.1). The spectrum of the companion object is noisier but shows a rather flat low continuum level with no important absorption features, characteristic of an $\mathrm{H}$ II region-dominated zone.

Fluxes and equivalent widths of the emission lines were measured using the SPLOT routine of the IRAF package. This task integrates all the flux in the line between two given limits and over a fitted local continuum. All the emission lines detected were corrected for reddening using the Seaton (1979) law and the corresponding value of $C(\mathrm{H} \beta)$ derived in the way we explained in Sect. 3.1. The $\mathrm{H} \beta$ line flux, $F(\mathrm{H} \beta)$ and the equivalent width of several lines $(\mathrm{H} \alpha, \mathrm{H} \beta, \mathrm{H} \gamma, \mathrm{H} \delta$ and [O III]) are given in Table 4 , as well as the reddeningcorrected line intensity ratios relative to $\mathrm{H} \beta$ and their uncertainties. Colons indicate uncertainties greater than $40 \%$. Note that the values obtained for the $\mathrm{H} \alpha$ equivalent width of \#1, \#2, IRAS $08339+6517$ and the companion galaxy using the spectra and the $\mathrm{H} \alpha$ image are similar to within the uncertainties.

\subsubsection{Physical conditions of the ionized gas}

We have studied the physical conditions and chemical abundances of the ionized gas from our 1-D spectra. Although the weak [O III] $\lambda 4363$ emission line is barely detected in the brightest galaxy, the underlying stellar absorption affecting $\mathrm{H} \gamma$ does not permit a proper measurement of that auroral line, except for the $12^{\prime \prime}$ aperture (see below). The oxygen abundance was derived then using the Pilyugin (2001) empirical calibration. The electron temperatures have been estimated from the $T$ ([O III]) and $T$ ([O II]) pairs that reproduce the total oxygen abundance obtained by applying the Pilyugin (2001) empirical method (see next section). Like Pilyugin, we have assumed a two-zone scheme, the usual relation $\mathrm{O} / \mathrm{H}=\mathrm{O}^{+} / \mathrm{H}^{+}+\mathrm{O}^{++} / \mathrm{H}^{+}$ and the linear relation between $T([\mathrm{O} \mathrm{III}])$ and $T([\mathrm{O} \mathrm{II}])$ based on photoionization models obtained by Garnett (1992). The finally adopted $T[\mathrm{O} \mathrm{III}]$ and $T[\mathrm{O} \mathrm{II}]$ for each burst are shown in Table 5. We have no determination of electron densities, so we assumed that $N_{\mathrm{e}}=100 \mathrm{~cm}^{-3}$ for all the objects.

The spectrum obtained using the $12^{\prime \prime}$ aperture allows a tentative determination of the electron temperature of the ionized gas because of the detection of the [O III] $\lambda 4363$ and [N II] $\lambda 5755$ emission lines. These weak auroral emission lines have an error of $\sim 54 \%$ and $34 \%$, respectively, giving $T([\mathrm{O}$ III $])=8900_{-1600}^{+1200}$ and $T([\mathrm{~N} \mathrm{II}])=9000_{-1300}^{+2600}$. These values are in agreement with those we obtained using the empirical calibration. Furthermore, the direct determination also agrees with the Garnett (1992) linear relation between the high and low electron temperatures.

\subsubsection{Abundance analysis}

Pilyugin (2001) performed a detailed analysis of observational data combined with photoionization models to obtain empirical calibrations for the oxygen abundance from the relative intensities of strong optical lines. He used both the $R_{23}$ and $P$ (an indicator of the hardness of the ionizing radiation) parameters for his calibration, the so-called $P$-method, which can be used in moderately high-metallicity $\mathrm{H}$ II regions $(12+\log \mathrm{O} / \mathrm{H} \geq 8.15)$. We have used this empirical calibration to derive the total oxygen abundance in IRAS 08339+6517 and its companion galaxy. We have also estimated the total oxygen abundance making use of the Denicoló et al. (2002) empirical calibration, which involves the [N II] $\lambda 6583 / \mathrm{H} \alpha$ ratio. The oxygen abundances obtained using both methods are shown in Table 5. However, the $\mathrm{O} / \mathrm{H}$ ratios obtained using the last calibration seem to be systematically higher than those obtained using the Pilyugin (2001) method, as we reported in a previous study (López-Sánchez et al. 2004b). Here, an offset of 0.15-0.20 dex is found between both calibrations. We prefer to consider the Pilyugin (2001) abundances as the more appropriate ones because his calibration take into account the excitation parameter of the ionizing radiation.

All the ionic abundances shown in Table 5 (except $\mathrm{He}^{+}$and $\mathrm{Fe}^{++}$) have been calculated using the IRAF NEBULAR task from the intensity of collisionally excited lines, assuming the $T$ ([O III]) for the high ionization potential ions $\mathrm{O}^{++}$and $\mathrm{Ne}^{++}$, and $T\left([\mathrm{O} \mathrm{II}]\right.$ ) for the low ionization potential ions $\mathrm{O}^{+}$and $\mathrm{N}^{+}$. We have assumed the standard ionization correction factor (icf) by Peimbert \& Costero (1969), N/O = $\mathrm{N}^{+} / \mathrm{O}^{+}$, to derive the nitrogen abundance of the objects. The $\mathrm{He}^{+} / \mathrm{H}^{+}$ratio has been derived from the He I $\lambda 5876$ line observed in each knot, using the predicted line emissivities calculated by Smith et al. (1996). 

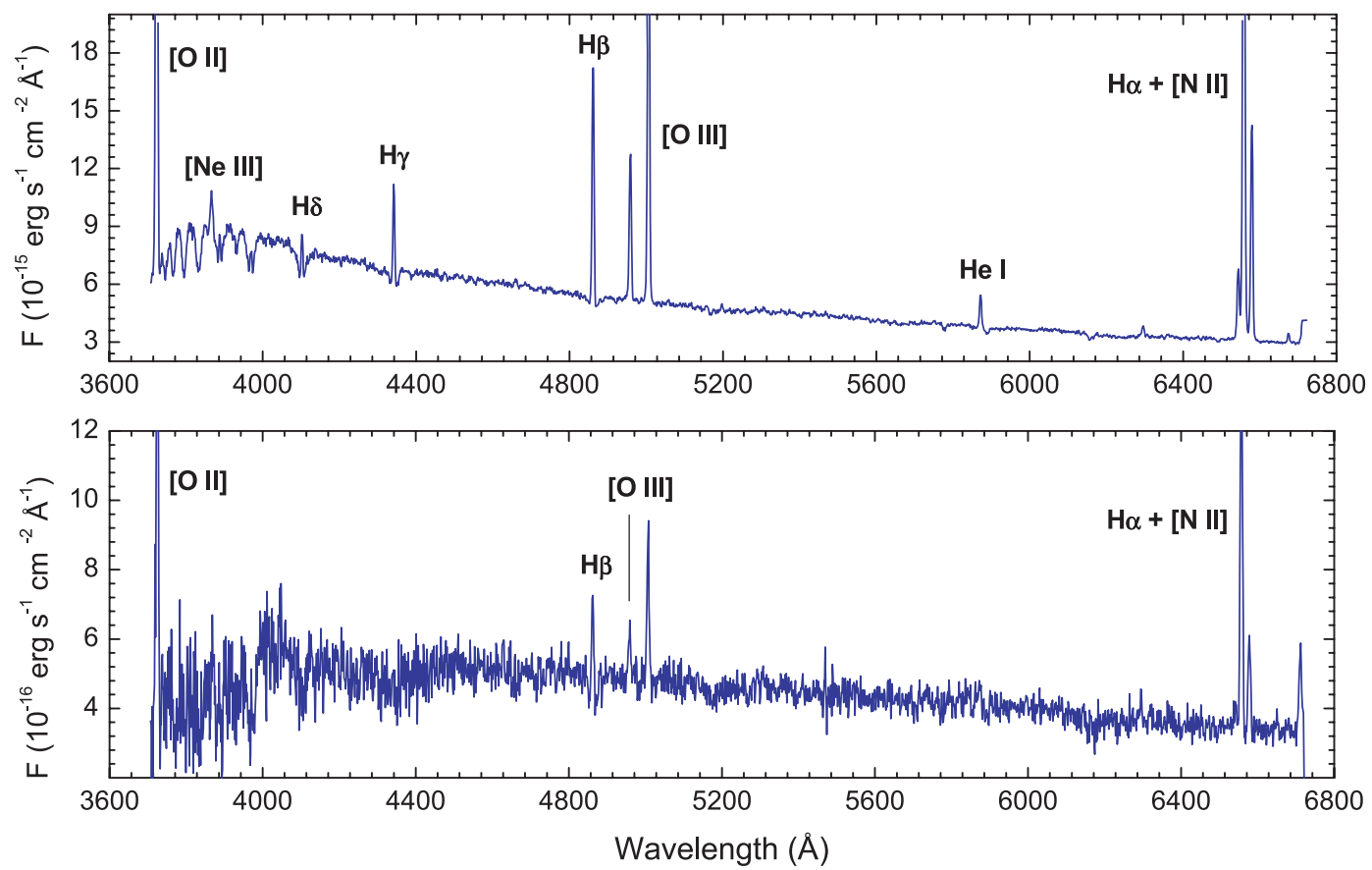

Fig. 4. Redshift-corrected spectra of IRAS 08339+6517 (top) and the companion galaxy 2MASX J08380769+6508579 (bottom). The most important emission lines are labeled. The spectra have been scaled down in flux in order to distinguish the faint lines. They are not corrected by reddening. Note the high continuum level and the H I absorptions due to the underlying stellar population in the spectrum of the main galaxy.

Table 5. Chemical abundances of the galaxies and analyzed knots inside IRAS 08339+6517.

\begin{tabular}{|c|c|c|c|c|c|c|}
\hline Object & IRAS 08339+6517 & $\# 1$ & $\# 2$ & A & B & Companion \\
\hline$T_{\mathrm{e}}[\mathrm{O} \text { III }]^{a}$ & 8700 & 7900 & 7900 & 8700 & 7700 & 9050 \\
\hline$T_{\mathrm{e}}[\mathrm{O} \mathrm{III}]^{b}$ & 9100 & 8500 & 8500 & 9100 & 8400 & 9300 \\
\hline $12+\log \mathrm{O} / \mathrm{H}^{c}$ & $8.45 \pm 0.10^{f}$ & $8.55 \pm 0.10$ & $8.53 \pm 0.10$ & $8.42 \pm 0.10$ & $8.58 \pm 0.10$ & $8.38 \pm 0.10$ \\
\hline $12+\log \mathrm{O} / \mathrm{H}^{d}$ & $8.71 \pm 0.20$ & $8.71 \pm 0.20$ & $8.70 \pm 0.20$ & $8.63 \pm 0.20$ & $8.72 \pm 0.20$ & $8.62 \pm 0.20$ \\
\hline$\left(\mathrm{O}^{++}+\mathrm{O}^{+}\right) / \mathrm{O}^{+}$ & 1.53 & 1.85 & 1.63 & 1.29 & 2.04 & 1.81 \\
\hline $\log \mathrm{N}^{+} / \mathrm{O}^{+}$ & -0.94 & -0.89 & -0.94 & -1.10 & -0.86 & -1.13 \\
\hline $12+\log \mathrm{N} / \mathrm{H}$ & 7.51 & 7.65 & 7.59 & 7.21 & 7.72 & 7.26 \\
\hline $12+\log \mathrm{Ne}^{++} / \mathrm{H}^{+}$ & 7.54 & 7.73 & 7.56 & $\ldots$ & $\ldots$ & 7.48: \\
\hline $12+\log \mathrm{He}^{+} / \mathrm{H}^{+}$ & $10.91 \pm 0.03$ & $10.98 \pm 0.06$ & $10.92 \pm 0.07$ & $\ldots$ & $\ldots$ & $10.90 \pm 0.13$ \\
\hline $12+\log \mathrm{Fe}^{++} / \mathrm{H}^{+}$ & $5.95 \pm 0.16$ & 5.91: & $\ldots$ & $\ldots$ & $\ldots$ & $\ldots$ \\
\hline$[\mathrm{O} / \mathrm{H}] /[\mathrm{O} / \mathrm{H}]_{\odot}^{e}$ & 0.62 & 0.78 & 0.74 & 0.58 & 0.83 & 0.52 \\
\hline
\end{tabular}

${ }^{a}$ Calculated using empirical calibrations of Pilyugin (2001).

${ }^{b} T_{\mathrm{e}}[\mathrm{OII}]$ calculated from empirical calibration and the relation given by Garnett (1992).

${ }^{c}$ Determined using the empirical calibration of Pilyugin (2001).

${ }^{d}$ Determined using the $[\mathrm{N} \mathrm{II}] / \mathrm{H} \alpha$ ratio (Denicoló et al. 2002).

${ }^{e}$ Asuming $12+\log [\mathrm{O} / \mathrm{H}]_{\odot}=8.66 \pm 0.05$ (Asplund et al. 2004).

${ }^{f}$ The oxygen abundance derived for this object using the direct method is 8.42 .

We have also corrected for collisional contribution following the calculations by Benjamin et al. (2002). Self-absorption effects were not considered for the determination of $\mathrm{He}^{+} / \mathrm{H}^{+}$ but only errors derived from the observed uncertainties in line measurement.

We have detected the [Fe III] $\lambda 4658$ emission line in the integrated spectrum of the main galaxy and in knot \#1. The calculations for $\mathrm{Fe}^{++}$have been done with a 34 level model-atom that uses the collision strengths of Zhang (1996) and the transition probabilities of Quinet (1996). Applying the relation given by Rodríguez \& Rubin (2005), we derived a total Fe abundance of $12+\log (\mathrm{Fe} / \mathrm{H})=6.11$ and $\log (\mathrm{Fe} / \mathrm{O})=-2.60$ for the galaxy.

In general, we find that the $\mathrm{O} / \mathrm{H}$ ratio of the knots inside IRAS $08339+6517$ are similar [around 8.5, in units of $12+\log (\mathrm{O} / \mathrm{H})]$, except object $\mathrm{A}$, which has a slightly lower value $[12+\log (\mathrm{O} / \mathrm{H})=8.42]$. On the other hand, the oxygen 


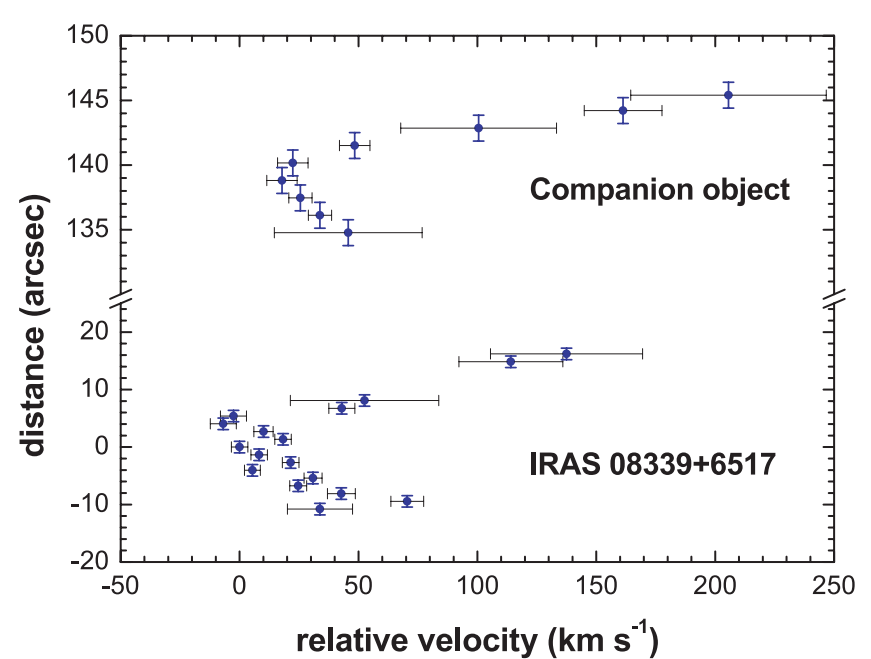

Fig. 5. Position-velocity diagram for the slit position observed, analyzed in $1.1^{\prime \prime}$ bins. The horizontal bars represent the uncertainty of the Gaussian fitting for each point. Note that the vertical axis has been broken for clarity. Northwest is up.

abundance of the companion galaxy is a bit lower than that derived for IRAS $08339+6517$. The $\mathrm{N}^{+} / \mathrm{O}^{+}$ratios are similar for all the objects, within the expected values for spiral and irregular galaxies with the same oxygen abundance (Garnett et al. 1999). These facts suggest that both galaxies have suffered a comparable degree of chemical enrichment despite their different absolute magnitudes.

\subsubsection{Kinematics of IRAS $08339+6517$}

The kinematics of the ionized gas were studied via the spatially resolved analysis of the $\mathrm{H} \alpha$ line profile along the slit position. We have extracted zones 6 pixels wide (1.1" long) covering all the extension of the line-emission zones. The Starlink DIPSO software (Howarth \& Murray 1990) was used to perform a Gaussian fit to the $\mathrm{H} \alpha$ profiles. For each single or multiple Gaussian fit, DIPSO gives the fit parameters (radial velocity centroid, Gaussian sigma, $F W H M$, etc.) and their associated statistical errors. In Fig. 5 we show the position-velocity diagram obtained, indicating the position of the studied objects. All the velocities are referred to the heliocentric velocity of the center of IRAS $08339+6517\left(5775 \mathrm{~km} \mathrm{~s}^{-1}\right)$.

The main galaxy has an apparent global rotation pattern, but it also shows local velocity fluctuations that could be produced by the effects of the very recent star formation activity (gas motions due to the combination of the winds of massive stars and supernova explosions) or by distortions in the gas associated with interaction effects (with the companion galaxy or, perhaps, with an hypothetical pre-existing external object that could have merged with IRAS $08339+6517$ in the past). Interaction features are clearly detected in the outer northwest area of IRAS $08339+6517$, where the ionized gas does not follow the general kinematics but presents a continuous variation between +45 and $+130 \mathrm{~km} \mathrm{~s}^{-1}$. These positions coincide with the $\mathrm{H} \alpha$ emission detected in knot B and the NW arm. The companion galaxy seems to shows an apparent rotation behavior in its southeast area (its brighter zone), although an important velocity gradient (around $+200 \mathrm{~km} \mathrm{~s}^{-1}$ ) is found in the side opposites the main galaxy. This fact suggests again that gravitational interactions have affected the dynamics of the ionized gas.

Assuming the global kinematics of the galaxies as circular rotation, we can make a rough estimate of their Keplerian mass, $M_{\text {kep }}$, taking into account the half of the maximum velocity difference in the galaxy and the half of the spatial separation corresponding to these maxima. We do not consider in this calculus the effect of the velocity gradients presented in both galaxies. We obtain masses of $(10 \pm 3) \times 10^{9} M_{\odot}$ and $(8 \pm 2) \times 10^{8} M_{\odot}$ for IRAS $08339+6517$ and its companion galaxy, respectively, assuming circular orbits, Keplerian dynamics and an inclination of $90^{\circ}$. We remark again that these values are only very tentative estimations of the masses of the galaxies.

We have compared the Keplerian mass, the neutral gas mass, $M_{\mathrm{HI}}$, and the ionized hydrogen mass, $M_{\mathrm{H} \mathrm{II}}$ (see Table 2) of the both galaxies and find substantial differences between them. IRAS $08339+6517$ has higher $M_{\mathrm{H} \mathrm{II}} / M_{\mathrm{HI}}$ and $M_{\mathrm{kep}} / M_{\mathrm{HI}}$ ratios (0.013 and 9.1, respectively), while the companion object has 0.0004 and 1.1. However, if we consider that the tidal H I gas found between both objects (Cannon et al. 2004), with a mass of $M_{\mathrm{HI}}=(3.8 \pm 0.5) \times 10^{9} M_{\odot}$, have been expelled from the main galaxy, the new $M_{\mathrm{kep}} / M_{\mathrm{HI}}$ ratio of IRAS $08339+6517$ is 2.0, similar to that found in the companion galaxy.

The light-to-mass ratios, $L_{B} / M_{\mathrm{kep}}$ and $L_{B} / M_{\mathrm{HI}}$, are also very different for both galaxies. The main object has $L_{B} / M_{\mathrm{HI}} \sim$ 61 , much higher than the typical values for spiral and irregular galaxies, that are between 1.6 and 11.2 (Bettoni et al. 2003). The companion galaxy has a value between those limits $\left(L_{B} / M_{\mathrm{HI}} \sim 4.2\right)$, similar to an Sbc galaxy. If we again consider that the tidal neutral gas belongs to IRAS $08339+6517$, the new light to neutral gas ratio is $\sim 13.7$, only a bit higher than the expected value for an Sa galaxy $(11.2 \pm 2.0)$. The light-to-cold dust ratio, $L_{B} / M_{\text {dust }} \sim 1.5 \times 10^{4}$, is also the expected one for young spirals (Bettoni et al. 2003). The mass of the dust was calculated using the FIR $60 \mu \mathrm{m}$ and $100 \mu \mathrm{m}$ flux densities (Moshir et al. 1990) together with the relation given by Huchtmeier et al. (1995, their last equation in p. 678). All these facts support the idea that the tidal $\mathrm{HI}$ gas has been formed from material mainly stripped from IRAS 08339+6517.

In order to improve our knowledge of the kinematics of the system, we have compared the position-velocity diagram obtained from our analysis of the $\mathrm{H} \alpha$ line profile in the optical spectrum with the position-velocity diagram obtained from H I data. We have used the Fig. 2 of Cannon et al. (2004) to extract the velocity of the neutral gas along our slit position (with PA $138^{\circ}$ ) in $10^{\prime \prime}$ steps. We referred all the H I velocities to the heliocentric velocity of the center of IRAS $08339+6517$ $\left(5775 \mathrm{~km} \mathrm{~s}^{-1}\right)$. In Fig. 6 we show the position-velocity diagram combining $\mathrm{H} \mathrm{I}$ and $\mathrm{H} \alpha$ data.

The general correspondence between both diagrams is rather good: the centers of the two galaxies and the kinematics of IRAS $08339+6517$ practically coincide in both neutral and ionized gas. Cannon et al. (2004) have remarked that, although the companion galaxy shows a component of solid-body rotation in neutral gas, the signs of rotation are less prominent in 


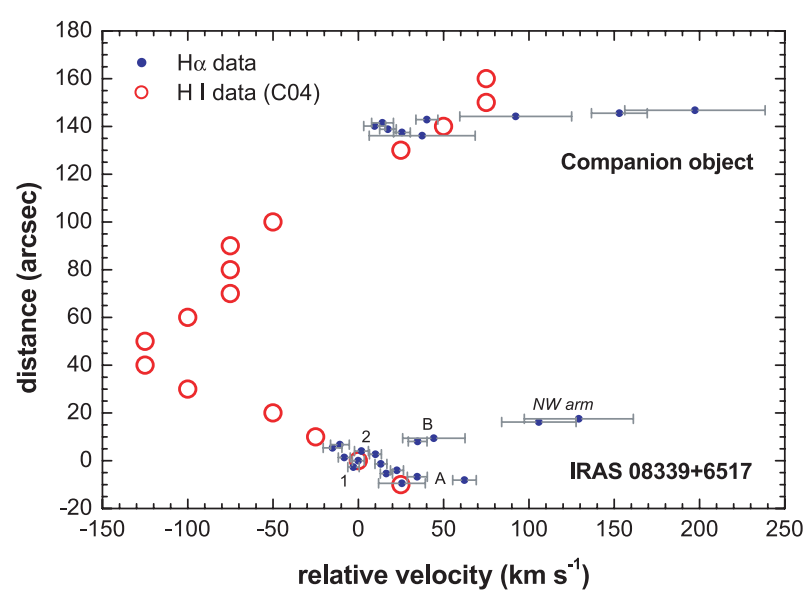

Fig. 6. Position-velocity diagram shown in Fig. 5 compared with that obtained using the H I data from Cannon et al. (2004) (open circles). The outflow of the neutral gas is clearly observed, as well as the decoupling of the ionized gas at the NW arm of IRAS $08339+6517$.

the main galaxy. But it could be masked in part by the outflow of the neutral gas in the direction of the observer, as is clearly observed in Fig. 6. This outflow of neutral gas was firstly suggested by González-Delgado et al. (1998) because of the excess absorption detected in the blue wing of the O VI $\lambda 1032$ profile. They also noted that the broadening of the Ly $\alpha$, Si II $\lambda 1260$ and C II $\lambda 1335$ absorption lines (the metallic lines are also resolved in two components) indicated large-scale motions of the interstellar gas, with a velocity even higher than $3000 \mathrm{~km} \mathrm{~s}^{-1}$. González-Delgado et al. (1998) suggested that the kinematics of the warm ionized interstellar medium are not driven by their gravitational potential but by the dynamical consequences of the violent star formation processes taking place in the galaxy. Kunth et al. (1998) attributed the existence of a secondary Ly $\alpha$ emission peak to the chaotic structure of the interstellar medium. Our $\mathrm{H} \alpha$ data suggest that, although these significant distorsions are found in the ionized gas, the kinematics of the galaxy still have an apparent important component of solidbody rotation.

However, we find a clear divergence between the kinematics of the neutral and the ionized gas at the NW arm of IRAS $08339+6517$, where object B is located: while the first has a velocity of $-50 \mathrm{~km} \mathrm{~s}^{-1}$ with respect to the center of the galaxy, the second shows a velocity around $+100 \mathrm{~km} \mathrm{~s}^{-1}$. Thus, the ionized gas seems to be decoupled from the expanding motion of the tidal tail of neutral gas. This suggests that the interaction that has created the H I tail is more complex that we thought. Knot B is specially interesting because it has the hightest oxygen abundance $(12+\log \mathrm{O} / \mathrm{H}=8.58)$, being the higher of all the spectroscopically observed regions (see Table 5).

\section{Discussion}

\subsection{Ages of the bursts and stellar populations}

\subsubsection{Ages from $W(\mathrm{H} \alpha)$}

One of the best methods of determining the age of the recent bursts of star formation is through the $\mathrm{H} \alpha$ equivalent width, $W(\mathrm{H} \alpha)$, since it decreases with time. We have used the STARBURST99 (Leitherer et al. 1999) spectral synthesis models to estimate the age of each knot, comparing the predicted $\mathrm{H} \alpha$ equivalent widths with the observed values. Spectral synthesis models with two different metallicities were chosen $\left(Z / Z_{\odot}=1\right.$ and 0.4 , the appropriate range of metallicities for the objects, see Table 5), both assuming an instantaneous burst with a Salpeter IMF, a total mass of $10^{6} M_{\odot}$ and a $100 M_{\odot}$ upper stellar mass. The ages obtained are compiled in Table 3 . All of them show a very young age for the last star formation episode, between 3.5 and $6 \mathrm{Myr}$, being the center of the galaxy (knot\#1) the youngest one. We can adopt an average age of 4-5 Myr for the most recent star formation episode in IRAS 08339+6517 and 5-6 Myr in its companion galaxy.

\subsubsection{Ages from evolutionary synthesis models}

González-Delgado et al. (1998) used evolutionary synthesis models to constrain the star formation history of IRAS $08339+6517$ from their UV spectra. They found that a 6-7 Myr burst with $M_{\text {up }} \geq 30 M_{\odot}$ or 9 Myr continuous star formation with $M_{\text {up }} \leq 30-40 M_{\odot}$ are compatible with their observations. They noted that the weakness of the stellar UV absorption lines and the small $\mathrm{H} \alpha$ equivalent width suggested significant dilution by an underlying stellar population. Our estimated ages from $W(\mathrm{H} \alpha)$ are a little lower than those they predicted. These authors also compared the C IV and Si IV profiles with those of the starburst galaxy NGC 1741, suggesting that IRAS $08339+6517$ is probably in a more advanced evolutionary stage. But the weakness of these lines could also be explained if the IMF is steeper or there exists a dilution of the stellar lines due to the underlying population. The latest study of NGC 1741 (member C in HCG 31) performed by López-Sánchez et al. (2004a) estimates an age of around 5 Myr and $E(B-V)=0.06$ for this galaxy. Thus, IRAS $08339+6517$ and NGC 1741 have very similar ages, but the first has a larger reddening contribution. Perhaps the effects of an underlying population is the most important factor in the dilution of the UV stellar lines in the González-Delgado et al. (1998) study.

We have compared our reddening-corrected colors with STARBURST99 (Leitherer et al. 1999) and PEGASE.2 (Fioc \& Rocca-Volmerange 1997) models to get an additional estimation of the age of the two galaxies. We have chosen these two models because while the firsts are based on Geneva tracks, the seconds use Padua isochrones (Bertilli et al. 1994) in which thermally pulsing asymptotic giant branch (TP-AGB) phases are included. We assumed an instantaneous burst with a Salpeter IMF, a total mass of $10^{6} M_{\odot}$ and a metallicity of $Z / Z_{\odot}=0.4$ and 1 for both models. In Fig. 7 we plot four different color-color diagrams comparing both STARBURST 99 and PEGASE. $2 Z / Z_{\odot}=0.4$ models with the observed values. We find a good correspondence using our optical values although PEGASE. 2 give older ages than STARBURST99. In particular, the $V-R$ color of IRAS $08339+6517$ implies an age of 40-60 Myr using STARBURST99 models, but 450-600 Myr when it is compared with PEGASE. 2 models. Furthermore, the NIR colors derived from 2MASS (reddening-corrected, see Table 2) are not in agreement with optical colors, specially in 

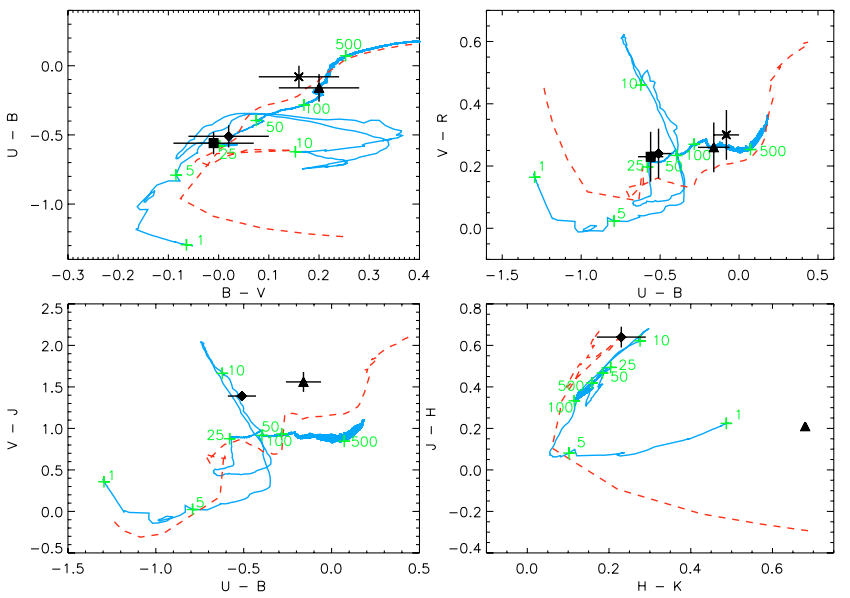

Fig. 7. Color-color diagrams showing the STARBURST99 (Leitherer et al. 1999) (blue/continuous line) and PEGASE.2 (Fioc \& Rocca-Volmerange 1997) (dashed/red line) models for an instantaneous burst with a Salpeter IMF and $Z / Z_{\odot}=0.4$ compared with our observed values for IRAS $08339+6517$ (circle) and its companion galaxy (triangle). We have also included the derived values for the underlying component in IRAS $08339+6517$ (cross) and the burst component (square). We indicate some age ticks (in Myr) along the plotted evolutionary tracks for the STARBURST99 model.

the case of the companion galaxy. The optical colors suggest an age of $\sim 30-50 \mathrm{Myr}$ for IRAS $08339+6517$ and older than $200 \mathrm{Myr}$ for its companion. The NIR colors imply ages older than 1-2 Gyr for both systems. The considerable difference in the age derived for the companion galaxy using the $W(\mathrm{H} \alpha)$ and the optical and NIR colors indicates that the old populations practically dominate the observed flux at these long wavelengths.

The fact that the ages derived from colors are older than those obtained from $W(\mathrm{H} \alpha)$ confirms that the effects of the underlying old population are important. The contribution of the old stellar population can be checked using the Stasińska et al. (2001) models of H II regions ionized by an evolving starburst embedded in a gas cloud of the same metallicity. We have chosen two models, both with metallicity $Z / Z_{\odot}=0.25$ and 1 and with a total mass of $10^{3}$ and $10^{6} M_{\odot}$. In Fig. 8 we plot our observational values of $W(\mathrm{H} \beta)$ and the [O III] $\lambda 5007 / \mathrm{H} \beta$ emission line flux (see Table 3) and compare them with the theoretical models. We find that all are located in the left area of the diagram, indicating a strong contribution of the underlying stellar continuum. Objects \#1 and \#2 show the best correspondence because they are regions mainly dominated by the recent star formation activity. The age derived for the objects following Fig. 8 is between 5 and $6 \mathrm{Myr}$, in agreement with those derived from $W(\mathrm{H} \alpha)$.

\subsubsection{Analysis of the surface brightness profiles}

In order to investigate the importance of older stellar populations we have performed an analysis of the surface brightness profiles of the two galaxies. We have taken concentric surfaces at different radii from the center of each system and

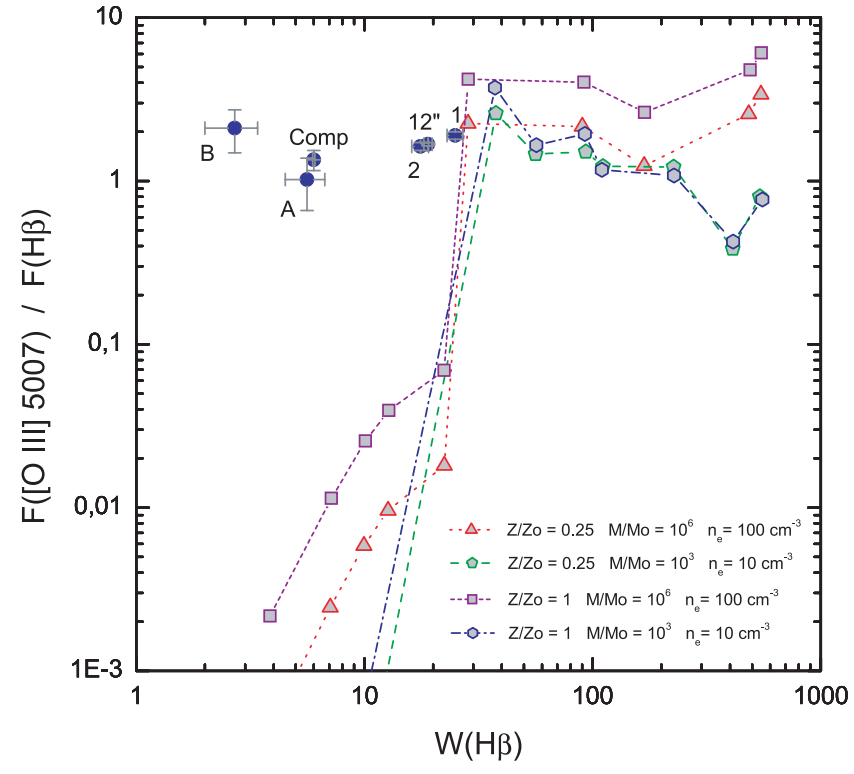

Fig. 8. $F[\mathrm{O} I I I] \lambda 5007$ versus $W(\mathrm{H} \beta)$ using the models by Stasińska et al. (2001). Tracks correspond to sequences of different metallicities and electronic densities. Each symbol marks the position of the models at $1 \mathrm{Myr}$ interval, starting in the upper-right corner of the diagram with an age of $1 \mathrm{Myr}$.

calculated the integrated flux inside each circle of area $A$ (in units of $\operatorname{arcsec}^{2}$ ). Then, the mean surface brightness inside this circle, $S B_{X}$ (in units of mag $\operatorname{arcsec}{ }^{-2}$ ), is derived using the relation:

$S B_{X}=m_{X}+2.5 \log A$,

being $m_{X}$ the magnitude in the filter $X$. The surface brightness, $\mu_{X}$, is the flux per square arcsec in the ring defined by two successive apertures. This simple technique is not adequate for the study of objects presenting irregular or complex morphologies (Cairós et al. 2001), but it is valid for approximately circular compact objects like IRAS $08339+6517$ and its companion galaxy. In Fig. 9 we show the surface brightness for $U$, $B, V$ and $R$ filters, $\mu_{U}, \mu_{B}, \mu_{V}$ and $\mu_{R}$, versus its radius, for IRAS $08339+6517$, whereas the surface brightness profiles for the companion galaxy are shown in Fig. 10. In both Figs. 9 and 10 we also include the radial color profiles $(U-B),(B-V)$ and $(V-R)$ derived by direct subtraction of light profiles.

In the main galaxy, the surface brightness profiles can be separated into two structures, indicating the existence of a low surface brightness component underlying the starburst that could be attributed to a disk-like structure inside the galaxy. We have performed an exponential law fitting to the profiles, following the expression:

$I=I_{0} \exp (-r / \alpha)$,

which describes a typical disk structure: $I_{\mathrm{O}}$ is the central intensity and $\alpha$ is the scale length. The fits were performed between $r_{\min }=14^{\prime \prime}$ and $r_{\max }=22^{\prime \prime}$ and are plotted over each profile with a dashed line. The upper limit was chosen in order to avoid contamination by the bright star at the SW of the galaxy (see Fig. 1); its effect on the surface brightness can be observed as 

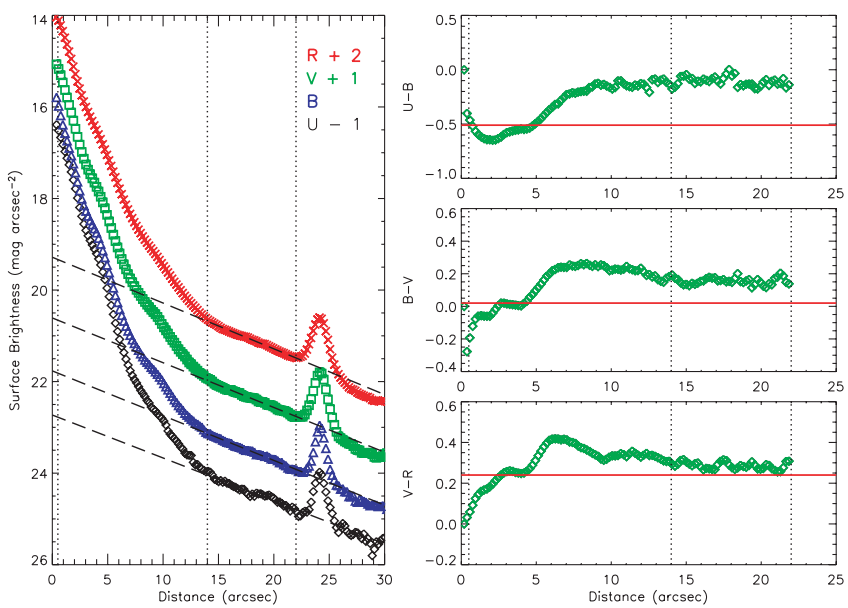

Fig. 9. Surface brightness and color profiles for IRAS $08339+6517$. The dashed lines in the surface brightness diagram represent exponential law fits to the profiles. The red horizontal lines in the color profile diagrams indicate the average color derived for each system. The dashed vertical lines indicate the radius of the seeing $\left(0.4^{\prime \prime}\right)$ and the interval used to perform the fit (between 14" and $22^{\prime \prime}$ ). The peak at $24^{\prime \prime}$ is consequence of the bright star located at the SW of the galaxy.

the peak at a radius of $24^{\prime \prime}$. Thus, it introduces an additional uncertainty to the linear fits to the underlying stellar emission. The fitting structural parameters are indicated in Table 6. Remark that an exponential intensity profile is typical not only for disks but also for low-mass spheroids, so it should not be taken as evidence that the 3D geometry of IRAS $08339+6517$ is approximated best by a disk. Note that the fit for the $U$ filter has highest error because the image is not deep enough to reach the faint surface brightness level needed to perform a good analysis. The fits can be used to determine the relative contribution of the underlying component and the burst (we will designate as burst the luminosity that can not be explained by the underlying component) to the luminosity at different radii. The relative contribution of the underlying component at several radii is also indicated in Table 6: it is only $2 \%$ at the central areas but equal to the contribution of the burst at $\sim 10^{\prime \prime}$. From $11^{\prime \prime}$ it dominates the total luminosity of the galaxy. The burst to total luminosity ratio is $\sim 85 \%$.

The variations of the radial color profiles also indicate the different stellar populations present in the main galaxy. Its central areas show bluer colors, but for radii $\geq 14^{\prime \prime}$ the color profiles have no gradients and show va/-lues redder to the ones derived from the integrated photometry. Surprising, we note a central color excess in the $U-B$ profile inside radius $\sim 2^{\prime \prime}$. It does not seem to be artificial because its size is larger than the seeing $\left(\sim 0.8^{\prime \prime}\right)$. In Fig. 11 we present an $U-R$ color map of IRAS $08339+6517$, as well as the $U-R$ color profile. The color map shows a ring-structure with bluer colors, that includes the two central bright bursts (see Fig. 2), around the nucleus of the galaxy. The $U-R$ color map also represents the dust distribution throughout the galaxy, that is somewhat inhomogeneous, as we previously note in Sect. 3.4.3. The nucleus of the galaxy seems to be dustier than its surroundings, being probably the
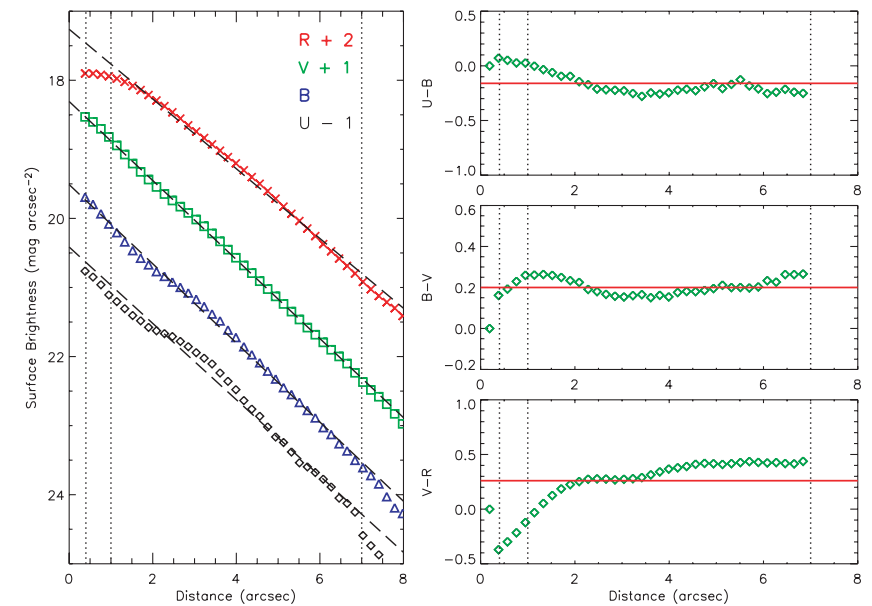

Fig. 10. Surface brightness and color profiles for the companion galaxy. The dashed lines in the surface brightness diagram represent exponential law fits to the profiles. The red horizontal lines in the color profile diagrams indicate the average color derived for each system. The dashed vertical lines indicate the radius of the seeing $\left(0.4^{\prime \prime}\right)$, and the interval used to perform the fit (between $2^{\prime \prime}$ and 7", the apparent optical radius of the galaxy derived from the deep optical images). Note the bump at $\sim 3.5^{\prime \prime}$ in $U$-filter, it is indicating the star-formation zone off-center this dwarf galaxy.

explanation of the central color excess observed in both $U-B$ (Fig. 9) and $U-R$ (Fig. 11) color profiles. This feature is not observed in the others color profiles because extinction in $U$ filter is much more effective.

We have estimated the colors of the underlying component and the burst using the fits to the light profiles derived above. They are plotted in Fig. 7. As we expected, the underlying component has significantly redder colors than the burst, supporting that it is composed by an older population. Their $U-B \sim-0.08$ and $B-V=0.16$ colors imply a minimal age between 200 and $300 \mathrm{Myr}$, although it rises to 700-900 Myr considering the $V-R=0.30$ color. For the burst component we find $U-B=-0.51, B-V=0.02$ and $V-R=0.23$, suggesting an age between 15 and $25 \mathrm{Myr}$ using both STARBURST99 and PEGASE. 2 models. These values are not as young as those obtained from $W(\mathrm{H} \alpha)$; it may indicate that, besides the disk-like underlying structure, which has been removed, an additional contribution of older stars also exists in the central areas of the galaxy.

On the other hand, the fits in the companion galaxy were performed between $r_{\min }=2^{\prime \prime}$ and $r_{\max }=7^{\prime \prime}$. They do not show two structural components, although deviations from the fits can be observed in the inner area and at $r \sim 3.5^{\prime \prime}$ (see Fig. 10) where the starburst is located and accounts for $\sim 10 \%$ of the surface brightness. It seems to be somewhat more evident in the profile derived for the $U$-filter. In general, the contribution of the starburst is diluted under the old stellar component, that accounts of $99 \%$ of the total luminosity of this dwarf galaxy. High spatial resolution photometry in NIR (Noeske et al. 2003) should be performed for a proper separation of these two stellar components. The radial color profiles are rather constants, with values similar to those derived from aperture photometry, also 
Table 6. Structural parameters of IRAS $08339+6517$ and its companion galaxy. The total luminosity in each band and the relative contribution of the underlying component at some radii are also shown.

\begin{tabular}{|c|c|c|c|c|c|c|c|c|c|c|}
\hline \multirow[b]{2}{*}{ Filter } & \multicolumn{5}{|c|}{ IRAS $08339+6517$} & \multirow[b]{2}{*}{$\begin{array}{c}\% \text { UC } \\
14^{\prime \prime} \\
\end{array}$} & \multirow[b]{2}{*}{$\begin{array}{c}\mu_{0} \\
\left(\text { mag }^{\prime \prime-2}\right) \\
\end{array}$} & \multicolumn{3}{|c|}{ Companion } \\
\hline & $\begin{array}{c}\mu_{0} \\
\left(\mathrm{mag}^{\prime \prime-2}\right)\end{array}$ & $\begin{array}{c}\alpha \\
(\mathrm{kpc}) \\
\end{array}$ & $\begin{array}{c}L_{\text {total }} \\
\left(10^{43} \mathrm{erg} \mathrm{s}^{-1}\right) \\
\end{array}$ & $\begin{array}{c}\% \text { UC } \\
r=2.5^{\prime \prime}\end{array}$ & $\begin{array}{c}\% \text { UC } \\
6^{\prime \prime}\end{array}$ & & & $\begin{array}{c}\alpha \\
(\mathrm{kpc})\end{array}$ & $\begin{array}{c}L_{\text {total }} \\
\left(10^{41} \mathrm{erg} \mathrm{s}^{-1}\right) \\
\end{array}$ & $\begin{array}{c}\% \text { UC } \\
r=3.5^{\prime \prime}\end{array}$ \\
\hline$U^{a}$ & 21.73 & 4.49 & $2.15 \pm 0.08$ & 1 & 13 & 92 & 19.41 & 0.76 & $7.1 \pm 0.5$ & 84 \\
\hline$B$ & 21.77 & 4.31 & $3.09 \pm 0.11$ & 2 & 17 & 99 & 19.51 & 0.73 & $14.0 \pm 0.6$ & 92 \\
\hline$V$ & 21.61 & 4.31 & $1.72 \pm 0.07$ & 3 & 16 & 96 & 19.31 & 0.74 & $9.2 \pm 0.5$ & 95 \\
\hline$R$ & 21.28 & 4.21 & $2.20 \pm 0.09^{b}$ & 3 & 14 & 96 & 19.26 & 0.83 & $11.9 \pm 0.6$ & 95 \\
\hline
\end{tabular}

${ }^{a}$ The errors in the fitting parameters in $U$ filter are higher than those found in the other filters. See text.

${ }^{b}$ Considering the $\mathrm{H} \alpha$ emission (see Table 3), the total luminosity in $R$ filter is $(2.08 \pm 0.10) \times 10^{43} \mathrm{erg} \mathrm{s}^{-1}$.
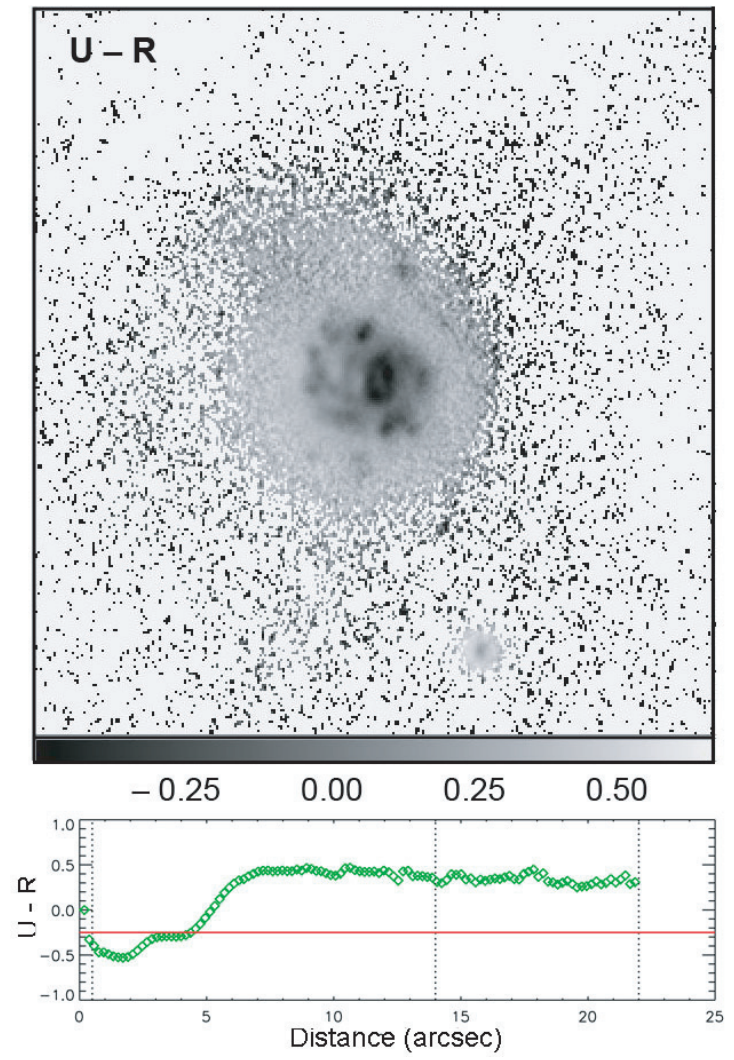

Fig. 11. $U-R$ color map of IRAS $08339+6517$, revealing a probable inhomogeneous distribution of the dust in the inner areas of the galaxy. Remark the blue ring-like structure around the nucleus of the galaxy. We also show the $U-R$ color profile for IRAS $08339+6517$. Note the color excess for radii $\leq 1^{\prime \prime}$. The red horizontal line indicate the average color, $U-R=-0.25$.

supporting the idea that the galaxy is mainly dominated by this old stellar population. Remember that the $U$ filter is not deep enough to reach a faint surface brightness level; its uncertainty is high for radii greatest than $r \sim 6^{\prime \prime}$.

\subsubsection{Ages from hydrogen stellar absorption lines}

The age of the stellar populations in a starburst galaxy can be estimated analyzing the equivalent widths of hydrogen and helium absorption lines. González-Delgado \& Leitherer (1999)
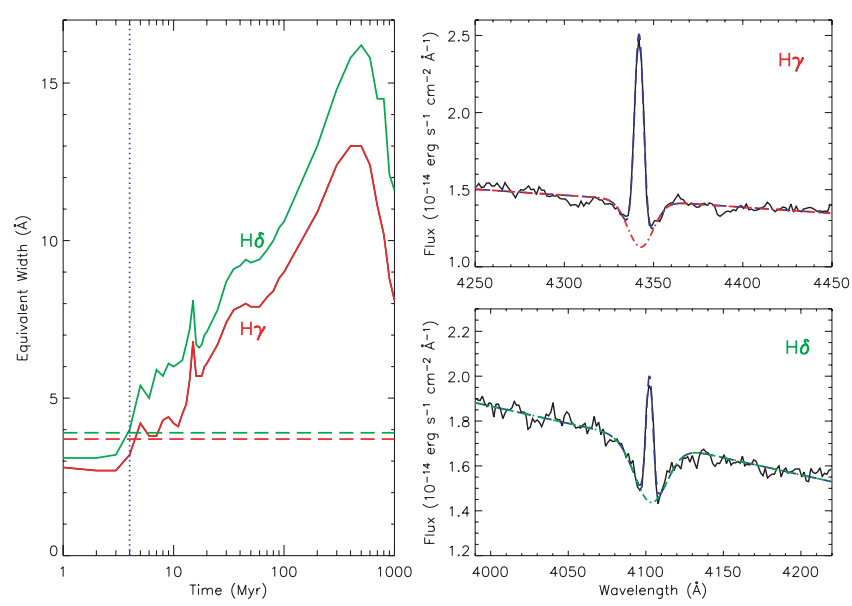

Fig. 12. (Right) $\mathrm{H} \gamma$ and $\mathrm{H} \delta$ emission lines of our $12^{\prime \prime}$ spectrum (black line) and a fit (long dashed line) using a narrow component ontop a broader absorption component (dash-dotted line). (Left) Variation of the equivalent widths of the $\mathrm{H} \gamma$ and $\mathrm{H} \delta$ absorption lines with the age of the burst, following the evolutionary stellar population synthesis models presented by González-Delgado et al. (1999). The dashed horizontal lines represent the equivalent widths derived from our spectrum. Note the decrement of the equivalent width for $t>500 \mathrm{Myr}$ in the models.

and González-Delgado et al. (1999) presented a synthetic grid of stellar H I Balmer and He I absorption lines in this kind of galaxies and developed evolutionary stellar population synthesis models for instantaneous bursts with ages between $1 \mathrm{Myr}$ and 1 Gyr, assuming a Salpeter IMF between 1 and $80 M_{\odot}$ and solar metallicity. The models indicate an increment in the equivalent widths with age. This analysis has been useful in similar studies of young starbursts or blue compact dwarf galaxies (e.g. Guseva et al. 2001).

We have used DIPSO software (Howarth \& Murray 1990) to perform a Gaussian fitting to the $\mathrm{H} \gamma$ and $\mathrm{H} \delta$ profiles of our $12^{\prime \prime}$ spectrum using a narrow Gaussian emission line profile ontop a broader absorption component to estimate their equivalent absorption widths. The fits are shown in Fig. 12. We have obtained $W_{\text {abs }}(\mathrm{H} \gamma)=3.7 \pm 0.1 \AA$ and $W_{\text {abs }}(\mathrm{H} \delta)=3.9 \pm 0.1 \AA$. Comparing these values with the González-Delgado et al. (1999) models, we estimate an age between 4 and 7 Myr for the stellar population. This age is surprising similar to the one derived from the $\mathrm{H} \alpha$ emission and not to that found from the 
analysis of the surface brightness profiles ( $>100 \mathrm{Myr})$. If the case of continuous star formation is considered instead of an instantaneous burst scenario, the values derived for $W_{\mathrm{abs}}(\mathrm{H} \gamma)$ and $W_{\text {abs }}(\mathrm{H} \delta)$ will imply a larger age, $\sim 15 \mathrm{Myr}$, but still younger that 100-200 Myr. González-Delgado et al. (1999) models also predict than the equivalent widths of Balmer lines in absorption decrease for $\sim 500 \mathrm{Myr}$, so the derived values might also imply ages $>1$ Gyr. It would be in agreement with the estimation given by NIR colors. The analysis of $\mathrm{H} \gamma$ and $\mathrm{H} \delta$ equivalent widths is not useful, in this case, to constraint the age of the underlying stellar population because the result merely is a luminosity-weighted age for the mixture of stars in the central part of the galaxy. At the center of IRAS $08339+6517$, the light is dominated by the young stars recently formed in the starburst, as we see both from the $\mathrm{H} \alpha$ image and the fact that it accounts for $\sim 85 \%$ of the total luminosity at this radius (the contribution of the underlying component is $\sim 15 \%$ at $r=6^{\prime \prime}$, see Table 6). In this sense, an appropriate study of the underlying stellar population using absorption lines would require to measure them at radii $r \geq 15^{\prime \prime}$, but our spectra are not able to reach these values.

\subsubsection{Ages from the spectral energy distribution}

Finally, we have employed spectral energy distributions (SED) to constraint the ages of the stellar populations. Although this method is dependent of the interstellar extinction, our estimation of the reddening contribution using the Balmer decrement (see Sect. 3.1) lets us to perform it avoiding the degeneracy problem between reddening and the ages of stellar populations. We have made use of the PEGASE. 2 code (Fioc \& Rocca-Volmerange 1997) to produce a grid of theoretical SEDs for an instantaneous burst of star formation and ages between 0 and $10 \mathrm{Gyr}$, assuming a $Z_{\odot}$ metallicity and a Salpeter IMF with lower and upper mass limits of $0.1 M_{\odot}$ and $120 M_{\odot}$. Although the grid include the ionized gas emission, we have neglected it because its contribution to the continuum is rather weak. In Fig. 13 we show our 12" extinction-corrected spectrum and the synthetic continuum spectral energy distributions derived assuming young ( $6 \mathrm{Myr}$ ) and old (140 Myr) ages. None of the individual synthetic spectra fitted our observed SED, so we constructed a model than combines $85 \%$ of the $6 \mathrm{Myr}$ model and $15 \%$ of the $140 \mathrm{Myr}$ model. We chosen these values following the relative contribution of the burst and the underlying component that we derived in the analysis of the surface brightness profiles at 6 " (see Table 6). We note that this combined model is in excellent agreement with the shape of our derreddened spectrum. However, we want to remark the degeneration existing in this kind of studies: several combinations of different relative contributions for old and young population models might also explain the observed spectrum. For example, a model which combines a contribution of 50\% for both $6 \mathrm{Myr}$ and $100 \mathrm{Myr}$ models also agrees with it.

The agreement between observed and synthetic spectrum is also found using the HI Balmer and He I absorption lines derived from González-Delgado et al. (1999) models. In Fig. 14 we plot our derredened normalized spectrum in the

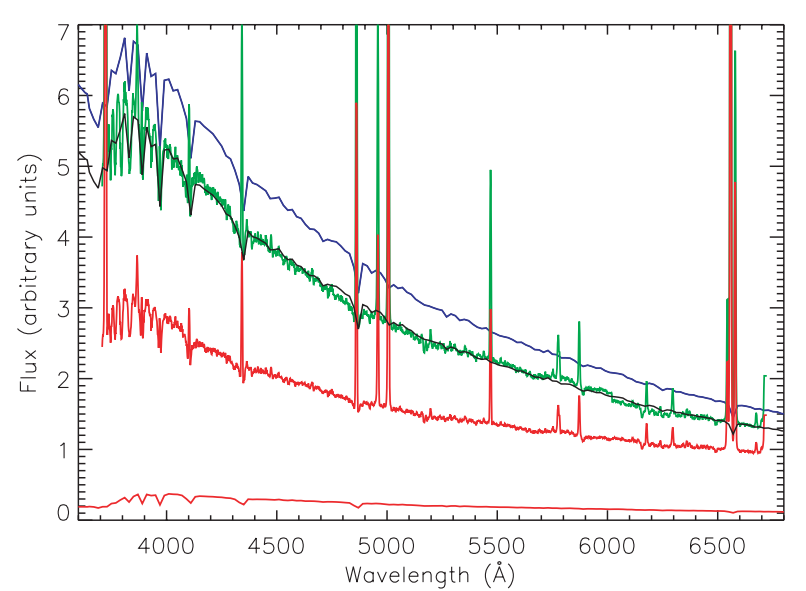

Fig. 13. Spectrum of IRAS $08339+6517$ compared with synthetic continuum spectral energy distributions obtained using the PEGASE.2 (Fioc \& Rocca-Volmerange 1997) code. The dotted line represents the observed spectrum uncorrected for extinction, whereas the gray/green continuous line is the extinction-corrected spectrum assuming $C(\mathrm{H} \beta)=0.22$. The upper continuous line corresponds to a model with an age of $6 \mathrm{Myr}$ (young population model), whereas the lower continuous one is a $140 \mathrm{Myr}$ model (old population model). The shape of our observed derredened spectrum fits with a model with a contribution of $85 \%$ for the young population and $15 \%$ for the old population is considered (continuous black line over the galaxy spectrum).

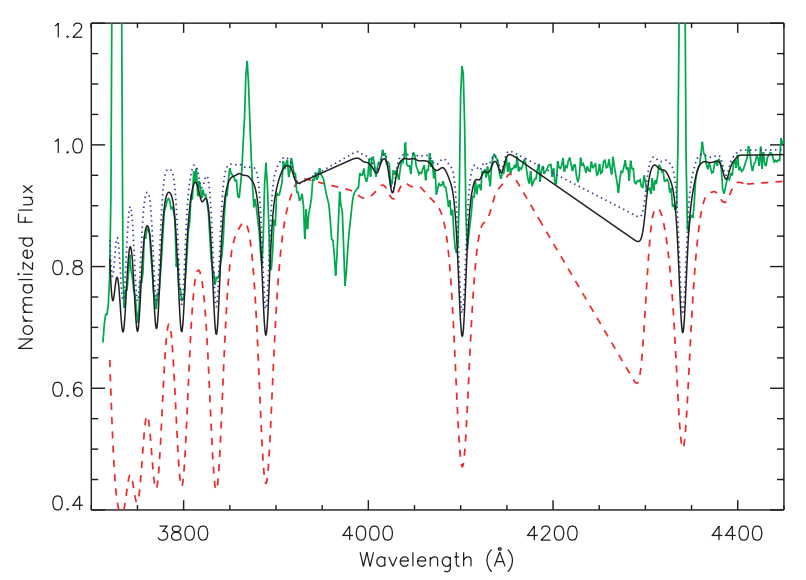

Fig. 14. Normalized dereddened spectrum of IRAS 08339+6517 (gray/green continuous line) compared with González-Delgado et al. (1999) models with 4 Myr (dotted line) and $200 \mathrm{Myr}$ (dashed line) at $Z_{\odot}$ metallicity. The best fit corresponds to a model with a contribution of $85 \%$ for the young population and $15 \%$ for the old population (continuous black line). Note that the models only give values for the position of the absorption lines and not for all wavelengths, being the reason of the straight lines presented between 3930 and $4000 \AA$ and 4150 and $4300 \AA$. The straight lines are artifacts connecting gaps in the dataset of the models.

3700-4450 ̊ range compared with their $Z_{\odot}$ models with ages of 4 and 200 Myr. The spectral resolution of the models were degraded to that of our observed spectrum. Again, a good fit is found when a combined model with a contribution of $15 \%$ for the old population and $85 \%$ for the young population is considered. 
Table 7. Star formation rates (SFR) derived for IRAS 08339+6517 using different relations (see Sect. 4.2). All the luminosities were calculated assuming a distance of $80 \mathrm{Mpc}$ for the galaxy.

\begin{tabular}{lccccc}
\hline \hline Range & Luminosity & Units & Value & SFR $\left(M_{\odot} \mathrm{yr}^{-1}\right)$ & Calibration \\
\hline X-ray & $L_{\mathrm{X}(0.2-2.0 \mathrm{keV})}$ & $\mathrm{erg} \mathrm{s}^{-1}$ & $2.81 \times 10^{41}$ & 61.8 & Ranalli et al. (2003) \\
Optical & $L_{\mathrm{H} \alpha}$ & $\mathrm{erg} \mathrm{s}^{-1}$ & $(12.0 \pm 0.6) \times 10^{41}$ & $9.5 \pm 0.5$ & Kennicutt (1998) \\
& $L_{B}$ & $L_{\odot}$ & $(6.61 \pm 0.24) \times 10^{10}$ & $1.92 \pm 0.07$ & Gallagher et al. (1984) \\
& $L_{B, U C}$ & $L_{\odot}$ & $(8.95 \pm 0.30) \times 10^{9}$ & $1.1 \pm 0.1$ & Calzetti (2001) \\
& $L_{[\mathrm{O} \mathrm{II}]}$ & $\mathrm{erg} \mathrm{s}^{-1}$ & $1.21 \times 10^{42}$ & 13 & Kennicutt (1998) \\
\multirow{4}{*}{ Far-Infrared } & & & & 8.9 & Kewley et al. (2004) \\
& $L_{\mathrm{FIR}}$ & $L_{\odot}$ & $5.63 \times 10^{10}$ & 9.7 & Kennicutt (1998) \\
& $L_{12 \mu \mathrm{m}}$ & $L_{\odot}$ & $1.78 \times 10^{10}$ & 11.6 & Roussel et al. (2001) \\
Radio & $L_{60 \mu \mathrm{m}}$ & $L_{\odot}$ & $3.90 \times 10^{10}$ & 9.2 & Condon (1992) \\
& $L_{1.4 \mathrm{GHz}}$ & $\mathrm{W} \mathrm{Hz}^{-1}$ & $2.57 \times 10^{22}$ & 6.4 & Condon et al. (2002) \\
\hline
\end{tabular}

In conclusion, the young stellar population in IRAS $08339+6516$ (the most recent starburst) has an age between 4 and $6 \mathrm{Myr}$, and it is located in the inner areas of the galaxy (radii $<6^{\prime \prime}$ ). It is superposed to a more evolved stellar population with an age not younger than 100-200 Myr, that fits a disk-like profile, and probably formed in early bursts. The NIR colors suggest that an older stellar population, with ages larger than 1-2 Gyr, also exists in IRAS $08339+6517$. Its companion galaxy is practically dominated by an old population with age $>250 \mathrm{Myr}$, although a recent starburst of around $6 \mathrm{Myr}$ is found in its external areas.

\subsection{The star formation rate}

We have derived the star formation rate (SFR) of IRAS 08229+6517 using empirical calibrations in different wavebands. The SFR is a key parameter for characterizing the formation and evolution of the galaxies. However, even in the well-observed galaxies of the local Universe, it remains quite uncertain because different methods yield different values. Much of this uncertainty is related to the unknown contribution of the dust obscuration in and around the star-forming regions.

One of the most extended methods of deriving the SFR is the use of hydrogen recombination line fluxes, especially the $\mathrm{H} \alpha$ flux. Since the flux in a hydrogen recombination line is proportional to the number of ionizing photons produced by the hot stars (which is also proportional to their birthrate), the SFR can be easily derived. The most recent calibration for starbursts is that derived by Kennicutt (1998). We have used this calibration to estimate the SFR in IRAS 08339+6517 from our $\mathrm{H} \alpha$ image, as we previously indicated in Sect. 3.3, obtaining $S F R_{\mathrm{H} \alpha}=9.5 M_{\odot} \mathrm{yr}^{-1}$ (see Table 7). Note that we have corrected the $\mathrm{H} \alpha$ flux for both reddening and [N II] contamination.

Many of the problems found in deriving the SFR from optical data can be avoided by measuring the far-infrared (FIR) and sub-millimeter spectral energy distribution. These are determined by the reradiation by the dust of energy absorbed in the visible and UV regions of the spectrum. Assuming that the dust completely surrounds the star forming regions, it acts as a bolometer reprocessing the luminosity produced by the stars. We have applied the Kennicutt (1998) correlation between the SFR and the FIR flux using the IRAS satellite data for IRAS $08339+6517, f_{60}=5.90 \mathrm{Jy}$ and $f_{100}=6.50 \mathrm{Jy}$ (Moshir et al. 1990), deriving $S F R_{\mathrm{FIR}}=9.7 M_{\odot} \mathrm{yr}^{-1}$. This value is in excellent agreement with the estimation derived from the $\mathrm{H} \alpha$ luminosity, indicating that the value for the extinction we have adopted seems to be fairly appropriate. We have also used others correlations between SFR and $60 \mu \mathrm{m}$ (Condon 1992) and $15 \mu \mathrm{m}$ (Roussel et al. 2001) luminosities (the last one assuming $L_{15 \mu \mathrm{m}} \sim L_{12 \mu \mathrm{m}}$ ), finding similar values (see Table 7).

The radio continuum flux can be also used as a star formation indicator. Nearly all of the radio luminosity from galaxies without a significant active galaxy nuclei (AGN) can be traced to recently formed massive $\left(M \geq 8 M_{\odot}\right)$ stars (Condon et al. 1992). Ten percent of the continuum emission at $1.4 \mathrm{GHz}$ is due to free-free emission from extremely massive main-sequence stars (thermal emission) and almost $90 \%$ is synchrotron radiation from relativistic electrons accelerated in the remmants of core-collapse supernovae (non-thermal emission). As the stars that contribute significantly to the radio emission have lifetimes $\tau \leq 3 \times 10^{7} \mathrm{yr}$ and the relativistic electrons have lifetimes $\tau \leq 10^{8} \mathrm{yr}$, the current radio luminosity is nearly proportional to the rate of massive star formation during the past $\tau \leq 10^{8} \mathrm{yr}$ (Condon et al. 2002).

We have estimated the SFR from the $1.4 \mathrm{GHz}$ luminosity for IRAS $08339+6517$ using the Condon et al. (2002) correlation. The $1.4 \mathrm{GHz}$ luminosity was derived from the 1.49 $\mathrm{GHz}$ luminosity (Condon et al. 1990) applying the relation between $L_{1.49 \mathrm{GHz}}$ and $L_{1.4 \mathrm{GHz}}$ given by Condon et al. (1991). The obtained value, $S F R_{1.4 \mathrm{GHz}}=6.4 M_{\odot} \mathrm{yr}^{-1}$, is a little lower than those derived from previous estimates but, as it was commented before, this rate corresponds to a larger time period than the $\mathrm{H} \alpha$ or FIR SFR indicators. Using the reddening-corrected $\mathrm{H} \alpha$ flux and the expression given by Dopita et al. (2002), we can also derive the thermal flux at $1.4 \mathrm{GHz}$ for the galaxy, $F_{1.4 \mathrm{GHz} \text { thermal }}=1.94 \mathrm{~mJ}$. It corresponds to around $6 \%$ of the total $1.4 \mathrm{GHz}$ flux, in agreement with the average value found in starburst galaxies (Condon 1992).

Assuming that $L_{B}=6.61 \times 10^{10} L_{\odot}$ and using the relation given by Gallagher et al. (1984) we derive a $S F R_{B}=$ $1.9 M_{\odot} \mathrm{yr}^{-1}$. Calzetti (2001) give a correlation between the $S F R$ 
of the starburst and the $B$ luminosity of the host galaxy. Using the structural parameters derived for IRAS 08339+6517 (see Table 6), we find that the total $B$-luminosity of the underlying component is $\sim 15 \%$ of the total, $L_{B, U C}=8.95 \times 10^{9} L_{\odot}$. Applying the Calzetti (2001) correlation, we derive $S F R_{B}=$ $1.1 M_{\odot} \mathrm{yr}^{-1}$. The $S F R$ derived from the blue luminosity corresponds to the past few billion years, whereas those derived from the $\mathrm{H} \alpha$ or FIR fluxes indicate the current $\left(<10^{7} \mathrm{yr}\right)$ SFR.

We can also use the relation given by Kennicutt (1998) between the SFR and the luminosity of [O II]. Using the $\mathrm{H} \alpha$ luminosity derived from our images for the entire galaxy (see Table 2) and the $[\mathrm{OII}] / \mathrm{H} \alpha$ ratio from our spectra $\left([\mathrm{O} \mathrm{II}] / \mathrm{H} \alpha=1.01\right.$ for the $12^{\prime \prime}$ aperture), we find $S F R_{[\mathrm{OII}]}=$ $13 M_{\odot} \mathrm{yr}^{-1}$, considering the low-limit of the Kennicutt (1998) calibration, corresponding to blue emission-line galaxies. A more realistic relation between the SFR and the luminosity of [O II], that take into account the oxygen abundance and ionization parameter of the ionized gas, was presented by Kewley et al. (2004) (their Eq. (15)). Applying this relation, we obtain $\left.S F R_{[\mathrm{O}} \mathrm{II}\right]=8.9 M_{\odot} \mathrm{yr}^{-1}$.

Global soft X-ray luminosity may seem to serve as an indicator for the SFR in star-forming galaxies. In the last years, several authors have tried to find a correlation between the soft $\mathrm{X}$-ray emission and the SFR. Using the Ranalli et al. (2003) expression and the $L_{\mathrm{X}}$ value from the ROSAT Satellite (Stevens \& Strickland 1998), $L_{0.2-2.0 \mathrm{keV}}=2.81 \times 10^{41} \mathrm{erg} \mathrm{s}^{-1}$, we derived $S F R_{L_{X}}=61.8 M_{\odot} \mathrm{yr}^{-1}$, a very high value compared with that found using the others calibrations. Stevens \& Strickland (1998) find that the X-ray luminosities of WR galaxies are considerably higher than those of the other galaxies with the same $B$ luminosities, consequence of the higher occurrence of superbubbles in WR galaxies. Superbubbles are hollow cavities with size of the order of $\mathrm{kpc}$, expanding with velocities between 25 and $150 \mathrm{~km} \mathrm{~s}^{-1}$ produced by the combination action of supernova explosions and stellar winds (Leitherer 1994). We can estimate the supernova rate, $v_{\mathrm{SN}}$, using the expression (7) in Kewley et al. (2000) that assumes a Salpeter IMF with lower and upper mass limits of 0.1 and $100 M_{\odot}$ and a minimum initial mass for supernova detonation of $8 M_{\odot}$ :

$v_{\mathrm{SN}}=7.42 \times 10^{-3} \times S F R$.

Assuming a $S F R=9.5 M_{\odot} \mathrm{yr}^{-1}$, we find a supernova rate of $v_{\mathrm{SN}} \sim 0.07 \mathrm{yr}^{-1}$, i.e., a supernova explosion each 14 years, a bit higher than the value usually found in starburst galaxies (around $0.02 \mathrm{yr}^{-1}$, Kewley et al. 2000). Thus, the relation given by Ranalli et al. (2003) between $S F R$ and the X-ray luminosity seems to be not suitable for very young starbursts.

The gas depletion timescale is defined as:

$\tau_{\text {gas }}=1.32 \times M_{\mathrm{HI}} / S F R$,

(Skillman et al. 2003), indicating the number of years that a galaxy may continue to form stars at its current rate. The factor 1.32 was introduced to account for He. For IRAS $08339+6517$ we obtain $\tau_{\text {gas }}=0.15$ Gyr $(0.68$ Gyr considering that the mass of the $\mathrm{H}$ I tidal tail). It is a very low value, indicating the starbursting nature of the galaxy.

In conclusion, the SFR derived using the $\mathrm{H} \alpha$, [O II], FIR, $15 \mu \mathrm{m}, 60 \mu \mathrm{m}$ and $1.4 \mathrm{GHz}$ luminosities are in a very good

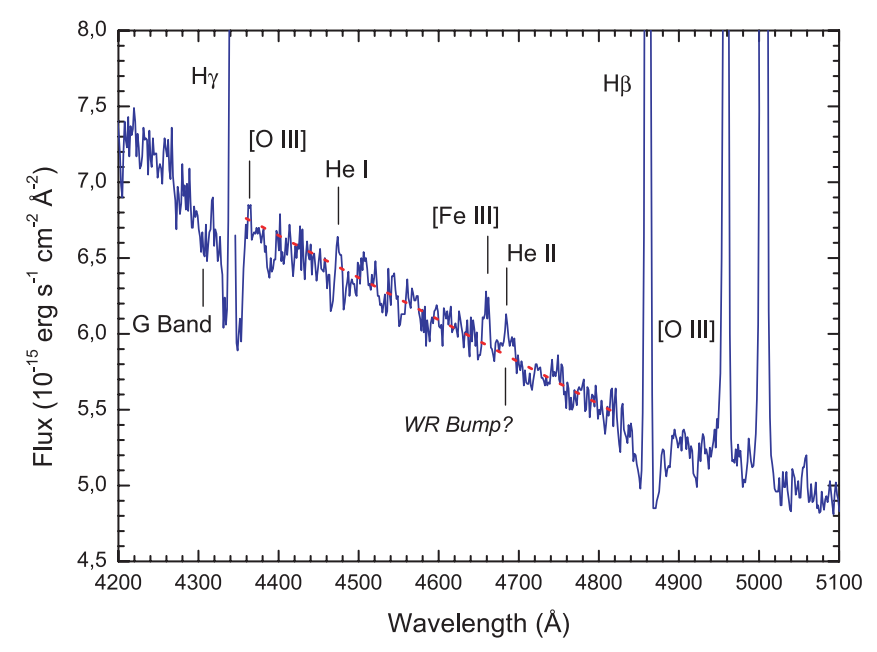

Fig. 15. Part of the spectrum of IRAS $08339+6517$ between 4300 and $5100 \AA$ showing the He II $\lambda 4686$ emission line associated with Wolf-Rayet stars. The dotted line indicates a linear fit to the continuum.

agreement and give a $S F R \sim 9.5 M_{\odot} \mathrm{yr}^{-1}$. The value of the $S F R$ found using the $B$-luminosity of the underlying population suggests that the star formation in the last $100 \mathrm{Myr}$ has not been as high as the present rate. Furthermore, the high SFR derived using X-ray luminosities implies a strong massive star formation in the last few Myr, supporting a high rate of supernova explosions and the possible detection of the WR stars.

\subsection{WR population}

IRAS $08339+6517$ was included in the X-ray study of WR galaxies performed by Stevens \& Strickland (1998) because previous authors (Conti 1991; González-Delgado et al. 1998) suggested that it has recently passed through the WR galaxy phase. However, our deep optical spectrum reveals that WR stars could still be present in the starburst. In Fig. 15 we show part of the spectrum of the central object of IRAS 08339+6517 (knot \#1) between 4200 and $5100 \AA$. It shows a very weak bump between 4660 and $4700 \AA$ and an emission line at $\lambda 4685.4$ that could correspond to the He II $\lambda 4686$ emission line. If this assumption is correct it would indicate the first detection of WR stars in this starburst galaxy. The UV spectrum of IRAS $08339+6517$ presented by Margon et al. (1988) showed a very weak emission line around $1640 \AA$ that could be attributed to He II $\lambda 1640$, line also associated with WR stars. However, these authors ruled this out because they do not detect the He II $\lambda 4686$ in their optical spectrum.

Although the He II $\lambda 4686$ emission line is weak, we have used the evolutionary synthesis models for O and WR populations in young starburst of Schaerer \& Vacca (1998) to perform a tentative estimation of the $\mathrm{WR} /(\mathrm{WR}+\mathrm{O})$ ratio. Assuming that all the contribution of the He II $\lambda 4686$ emission line comes from WNL stars and considering a luminosity of $L($ WNL 4686$)=1.7 \times 10^{36} \mathrm{erg} \mathrm{s}^{-1}$ for a WNL star (Vacca \& Conti 1992), we find around 310 WNL stars in this burst. To derive the $\mathrm{WR} /(\mathrm{WR}+\mathrm{O})$ ratio, the contribution of the WR stars to the total ionizing flux must be considered to 


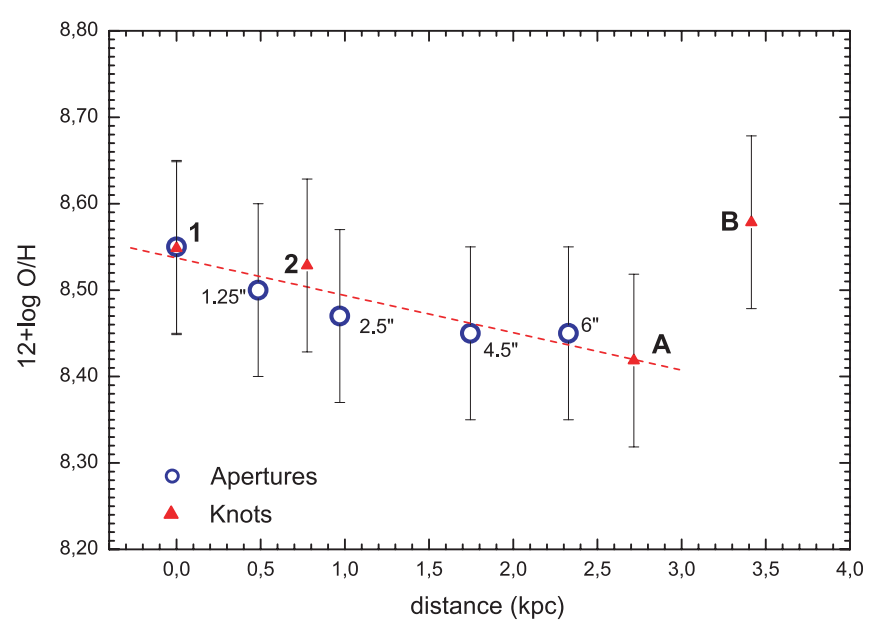

Fig. 16. Relation between metallicity and distance from the center of IRAS $08339+6517$ for the observed knots (triangles) and different aperture sizes (open circles). We have considered the oxygen abundances derived using the calibration by Pilyugin (2001). The dotted line is a linear fit to all the data except knot B, which shows the higher $\mathrm{O} / \mathrm{H}$ value. The linear fit suggests an abundance gradient along the disk.

obtain the total number of $\mathrm{O}$ stars. Assuming a luminosity of $L(\mathrm{H} \beta)=4.76 \times 10^{36} \mathrm{erg} \mathrm{s}^{-1}$ for a O7V star (Vacca \& Conti 1992) and $\eta \equiv \mathrm{O} 7 \mathrm{~V} / \mathrm{O}=0.25$ for an age of around 4.5 Myr (Schaerer $\&$ Vacca 1998), we derive around 10700 O stars. This implies a $\mathrm{WR} /(\mathrm{WR}+\mathrm{O})$ ratio of 0.03 in knot \#1. An identical value is found using the calibration between the $\mathrm{WR} /(\mathrm{WR}+\mathrm{O})$ ratio and the flux of the WR bump given by Schaerer \& Vacca (1998, their Eq. (17)).

The weak He II $\lambda 4686$ emission line is only detected in the spectra of knot \#1 ( $1^{\prime \prime}$ aperture) and in the 2.5" aperture but it is not found in any of the other spectra of the same galaxy. We suspect that maybe knot \#1, which hosts the youngest and most powerful burst in the system, is the only area where a substantial population may be present. Thus, aperture effects and the position of the slit can play an important role in the detection of WR features, as some authors have previously pointed out (Huang et al. 1999; López-Sánchez et al. 2004a,b).

However, although the He II $\lambda 4686$ emission line seems to really exist in knot \#1, deeper spectroscopical data will be necessary to confirm that it is broad and/or that the WR bump undoubtedly exists. Only under these facts the classification of IRAS $08339+6517$ as Wolf-Rayet galaxy can be established beyond any doubt.

\subsection{The nature of IRAS $08339+6517$}

\subsubsection{Radial dependence of metallicity and reddening}

We have analyzed the radial dependence of the derived metallicity of the eight apertures traced in IRAS 08339+6517 (four apertures corresponding with knots and four apertures with different sizes and centered on the nucleus, see Fig. 3), which is shown in Fig. 16. The oxygen abundance obtained from the empirical calibration of Pilyugin (2001) (see Tables 5 and 8) was assumed for each aperture.
Table 8. Principal properties derived for the ionized gas in IRAS 08339+6517 using different aperture sizes.

\begin{tabular}{cccc}
\hline \hline Aperture $\left(^{\prime \prime}\right)$ & $C(\mathrm{H} \beta)$ & $W_{\text {abs }}(\mathrm{HI})(\AA)$ & $12+\log (\mathrm{O} / \mathrm{H})$ \\
\hline $1 \times 1$ & $0.30 \pm 0.02$ & $1.1 \pm 0.1$ & $8.55 \pm 0.10$ \\
$2.5 \times 1$ & $0.26 \pm 0.02$ & $1.4 \pm 0.1$ & $8.50 \pm 0.10$ \\
$5 \times 1$ & $0.24 \pm 0.02$ & $1.5 \pm 0.1$ & $8.47 \pm 0.10$ \\
$9 \times 1$ & $0.21 \pm 0.02$ & $1.6 \pm 0.1$ & $8.45 \pm 0.10$ \\
$12 \times 1$ & $0.22 \pm 0.02$ & $1.8 \pm 0.1$ & $8.45 \pm 0.10$ \\
\hline
\end{tabular}

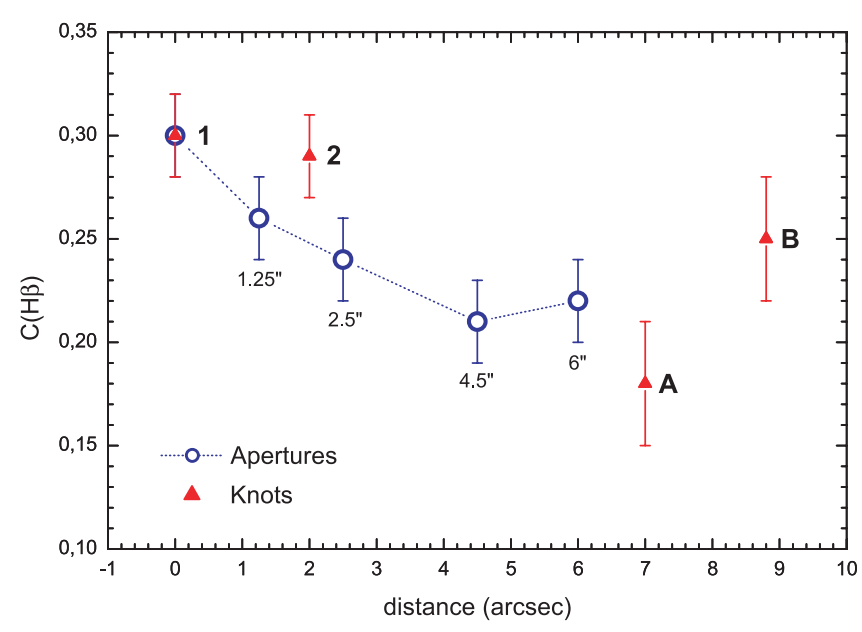

Fig. 17. Relation between the reddening coefficient, $C(\mathrm{H} \beta)$, with distance from the center of IRAS 08339+6517. Observed knots are plotted with triangles and the different aperture sizes with open circles connected with a dotted line.

Except for knot B, the oxygen abundances derived from the nucleus [knot \#1 with $12+\log (\mathrm{O} / \mathrm{H})=8.55]$ to the outer regions [knot A with $12+\log (\mathrm{O} / \mathrm{H})=8.42$ ] is continuously decreasing. Thus, despite the uncertainties, a possible presence of a weak abundance gradient along the disk of IRAS $08339+6517$ (around $-0.043 \mathrm{dex} \mathrm{kpc}^{-1}$ ) is detected. The average gradient in spiral galaxies is around $-0.06 \mathrm{dex} \mathrm{kpc}-1$ (Zaritski et al. 1994).

Besides the oxygen metallicity, the $W_{\text {abs }}$ calculated from the Balmer decrement increases with the aperture size, confirming again the existence of an evolved underlying stellar population. The $C(\mathrm{H} \beta)$ derived for different apertures inside IRAS $08339+6517$ also indicate an apparent slight gradient between the nucleus and the external zones of the galaxy (see Fig. 17). This may explain the differences found by González-Delgado et al. (1998) in the color excess between two different aperture sizes. For the nucleus, they estimated $E(B-V)=0.19$ (from the spectrum obtained with a $1.7^{\prime \prime} \times 1.7^{\prime \prime}$ aperture using the Goddard High-Resolution Spectrograph on the Hubble Space Telescope), similar to the value we derive for our $1^{\prime \prime} \times 1^{\prime \prime}$ aperture, $E(B-V)=0.20 \pm 0.01[C(\mathrm{H} \beta)=0.30 \pm$ $0.02]$. For the external zones, they derived $E(B-V)=0.10$ (using the $12^{\prime \prime}$ circular aperture with the Hopkins Ultraviolet Telescope), which corresponds with the one we obtained for our $12^{\prime \prime} \times 1^{\prime \prime}$ aperture, $E(B-V)=0.12 \pm 0.01[C(\mathrm{H} \beta)=0.22 \pm 0.02]$. The effect of the aperture size on derived integrated properties of galaxies has been recently studied by Kewley et al. (2005), 
who concluded that the difference between the nuclear and global derived values for metallicity, extinction and SFR is substantial: for example, for a late-type spiral galaxy, the metallicity of the nucleus is $\sim 0.14$ dex greater than the global metallicity of the galaxy.

\subsubsection{Knot B: a TDG or the remnant of a merger?}

As it can be observed in Fig. 16, the external knot B, located $3.4 \mathrm{kpc}$ from the center of IRAS $08339+6517$, shows a high metallicity $[12+\log (\mathrm{O} / \mathrm{H})=8.58]$, even a bit higher than that derived for the nucleus of the galaxy. Note that knots A and B are at rather similar distances from the nucleus but are located on opposite sides of the galaxy (see Fig. 2). Their spectra also show approximately the same $\mathrm{S} / \mathrm{N}$. Knot A seems to be a bright $\mathrm{H}$ II region (or a complex of H II regions) in the outer areas of the disk. Furthermore, as we can see in Sect. 3.4.3, knot B also shows a decoupled kinematics with respect to the motion of ionized gas associated to the tidal tail, so it might not be symply an intense star-formation region in the outskirts of the disk. We consider two possible explanations to these facts:

1. TDG nature. Dwarf objects showing high metallicities and decoupled kinematics are characteristics of candidate tidal dwarf galaxies, TDGs (Duc et al. 2000). The $B$-magnitude derived for this region is $m_{B} \sim 20.1$, implying an absolute $B$-magnitude of $M_{B} \sim-14.4$. Thus, considering its oxygen abundance, this object is away from the relation given by Richer \& McCall (1995) for dwarf irregular galaxies. Following the analysis performed by Weilbacher et al. (2003), it means that knot B (perhaps, even all the NW arm, knot \#7 in Fig. 2) could be a TDG candidate. The mean metallicity of the objects in the Weilbacher et al. (2003) sample is $12+\log (\mathrm{O} / \mathrm{H})=8.34 \pm 0.14$, but at least five have oxygen abundances higher than 8.55 (see their Fig. 3), similar to the one derived for knot B. However, a genuine TDG must be a self-gravitating entity. We can not derive it from our spectrum, so it is not possible to confirm that B is really a TDG candidate made from material stripped from the internal regions of the galaxy.

2. Merger nature. Knot $B$ could be a remnant of a previous merging process suffered by IRAS $08339+6517$ in the past. It would explain the disturbed morphology in its outer regions, specially the long arc of material connecting the north of the galaxy with the southern bright ray (see Fig. 1). This scenario was previously suggested by Cannon et al. (2004) and would also account for the intensive star-formation activity throughout the galaxy. If this early merger really happened, it would be in a very advanced state. Following the detailed analysis of a galactic merger sequence presented by Hibbard \& Gorkom (1996), late-stage mergers have tidal appendages emanating from a single nucleus surrounded by a mostly relaxed stellar profile. Their deep $R$ image of NGC 3921, prototype of this stage of merging, has morphological signatures similar to those found in IRAS $08339+6517$. Other interesting minor merger is the Atoms-for-Peace galaxy, NGC 7252: Hibbard et al. (1994) found that most of its H I mass resides outside the merger remnant, also similar to the case of IRAS $08339+6517$. However, if the merger hypothesis is right, the interaction with the companion galaxy would not be necessary and both the optical plume and the detected H I tidal tail (see Fig. 18) would be aligned to the direction of the companion galaxy only by chance. The effects of the interaction with the external companion galaxy can not be discarded. Although a minor merger occurred at early times the interaction with the external object seems to be more important at the present time.

\subsubsection{The non-AGN nature of IRAS $08339+6517$}

IRAS $08339+6517$ does not host an active galactic nucleus $(\mathrm{AGN})$. Several facts support this statement:

1. its spectrum, which is similar to the ones of typical starburst galaxies;

2. the $F W H M$ of the emission lines. For example, the $F W H M$ of $\mathrm{H} \beta$ and [O III] $\lambda 5007$ are, corrected for instrumental broadening, $263 \mathrm{~km} \mathrm{~s}^{-1}$ and $259 \mathrm{~km} \mathrm{~s}^{-1}$, respectively; the typical FWHM median value range for AGNs is between 350 and $550 \mathrm{~km} \mathrm{~s}^{-1}$ (Véron et al. 1997);

3. the position of the observational data over the Dopita et al. (2000) diagnostic diagrams, that are consistent with the loci of typical H II regions and not with AGN;

4. its logarithmic ratio of FIR to radio flux density, $q=2.34$, that is consistent with the values derived for normal galaxies, $q=2.3$ (Condon et al. 1992);

5. and the correlation between FIR and radio emission that satisfied the galaxy, both using the FIR and $1.49 \mathrm{GHz}$ luminosities (Condon et al. 1991) or the $1.4 \mathrm{GHz}$ radio continuum and $60 \mu \mathrm{m}$ FIR fluxes (Yun et al. 2001).

\subsubsection{A luminous compact blue galaxy}

The properties observed in IRAS 08339+6517 suggest classifying it as a luminous compact blue galaxy (LCBG). LCBGs are $\sim L^{\star}\left(L^{\star}=1.0 \times 10^{10} L_{\odot}\right), M_{B}<-18.5$, blue $(B-V<$ $0.6)$, high mean surface brightness within the half-light radius $\left(S B_{B}<21\right.$ mag $\left.\operatorname{arcsec}^{-2}\right)$, vigorous starbursting galaxies with an underlying older stellar population (Guzmán et al. 1998). Considering the $B$ magnitude derived for IRAS $08339+6517$, we found that it satisfies all these characteristics: it has $(B-$ $V)=0.05 \pm 0.08, M_{B}=-21.58 \pm 0.04$ and for $2.6^{\prime \prime}$, its half-light radius in the $B$-band, it possesses a mean surface brightness of $S B_{B} \sim 17.1 \mathrm{mag} \operatorname{arcsec}^{-2}$.

LCBGs are not common at low redshifts (Guzmán et al. 2003) and their evolution and nature is still discussed: whereas Koo et al. (1995) and Guzmán et al. (1996) suggested that LCBGs are the progenitors of local low-mass spheroidal or irregular systems experiencing a strong starburst phase, Phillips et al. (1997) and Hammer et al. (2001) pointed out that they are the progenitors of present-day bulges of massive spirals. In the local Universe, LCBGs seem to be more evolved objects and even they could be the equivalent of the high $z$ Lymanbreak galaxies (Erb et al. 2003), being ideal templates for studies of galaxy evolution and formation. A recent sample of 


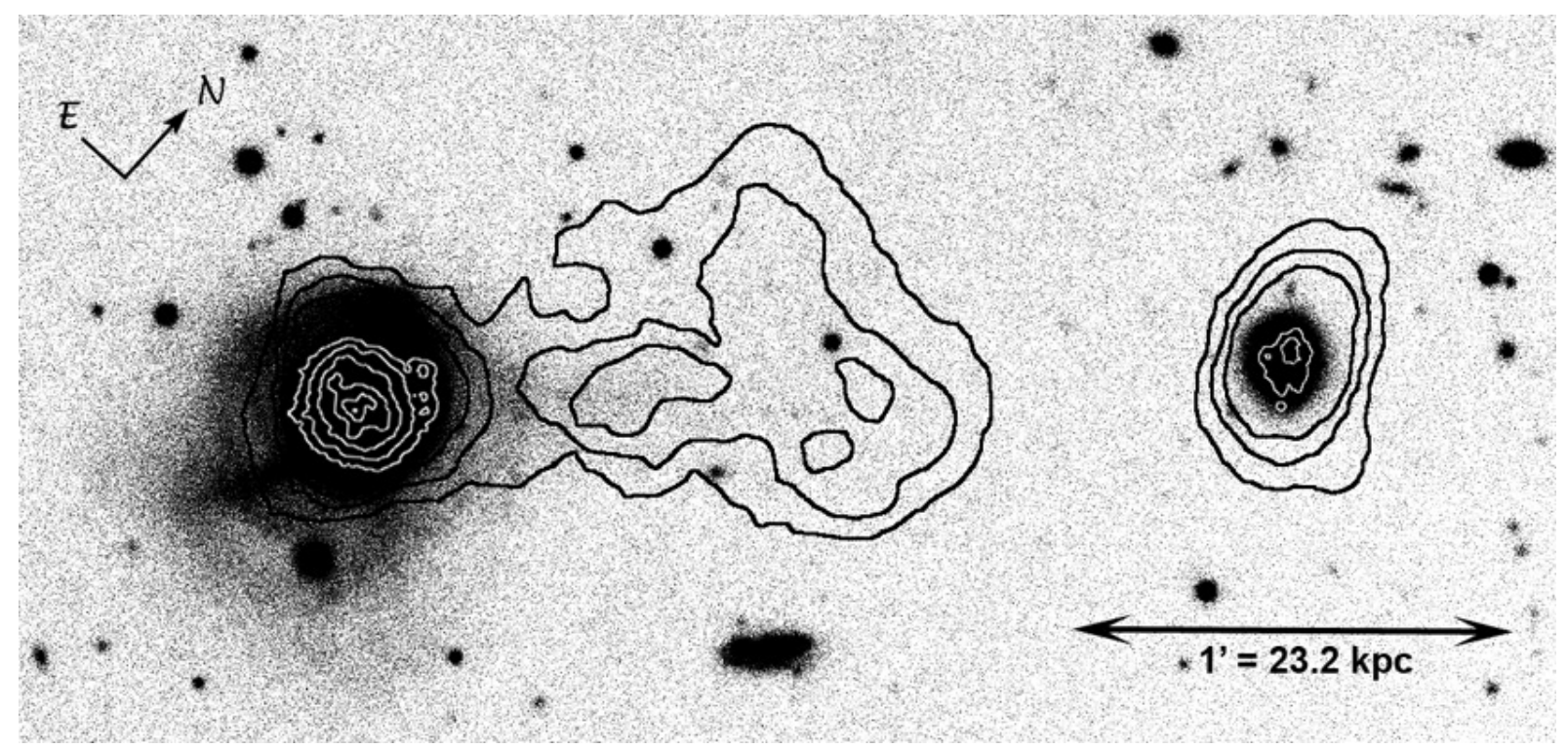

Fig. 18. Deep $R$ image of IRAS $08339+6517$ and its companion galaxy showing the faintest details. Our continuum-subtracted $\mathrm{H} \alpha$ image (white contours) and the H I map obtained by Cannon et al. (2004) (black contours) is superposed. Note that the weak optical plume in the direction of the companion galaxy coincides with the H I tidal tail.

LCBGs in the local Universe was presented by Werk et al. (2004) who found that, on average, LCBGs show strong starformation activity, emit detectable radio continuum flux and have lower metal abundances that those expected from the luminosity-metallicity relation for starbursting emission-line galaxies given by Melbourne \& Salzer (2002). All these properties are also satisfied by IRAS 08339+6517 (the Melbourne $\&$ Salzer (2002) relation gives an oxygen abundance of $\sim 9.2$, much higher than that we derived, $12+\log (\mathrm{O} / \mathrm{H})=8.45 \pm 0.10)$.

\subsubsection{The nature of the dwarf companion galaxy}

The dwarf companion galaxy follows the relation given by Richer \& McCall (1995) between absolute $B$-magnitude and oxygen abundance for dwarf irregular galaxies, indicating that it is not a TDG formed from material striped from IRAS $08339+6517$ but an external and independent dwarf galaxy. The apparent component of solid-body rotation found in $\mathrm{HI}$, the indications that the HI gas of the tidal tail has been probably stripped from the main galaxy and the lower star-formation activity found in the companion galaxy suggest that the effects of the interaction are not as intense as in IRAS 08339+6517.

\section{Conclusions}

We have used deep optical and $\mathrm{H} \alpha$ imagery together deep optical intermediate-resolution spectroscopy to analyze the morphology, colors, ages, stellar populations, physical conditions, kinematics and chemical abundances of the galaxy IRAS $08339+6517$ and its dwarf companion galaxy, 2MASX J08380769+6508579. Our data reinforce the results of the HI observations performed by Cannon et al. (2004) that both objects are in interaction and that the HI tidal tail seems to be formed mainly by material stripped from IRAS $08339+6517$.

We have obtained the oxygen abundances of both galaxies using empirical calibrations. The $\mathrm{O} / \mathrm{H}$ and $\mathrm{N} / \mathrm{O}$ ratios of the two galaxies are rather similar, suggesting a similar degree of chemical evolution of the system. We have analyzed the chemistry of the ionized gas using different aperture sizes and found an apparent metallicity gradient between the nucleus and the external zones of IRAS $08339+6517$. The reddening coefficient also shows differences between the central and external zones.

IRAS 08339+6517 shows important $\mathrm{H} \alpha$ emission in its inner regions. IRAS $08339+6517$ is not powered by an AGN but it is a nuclear starbursting galaxy. We have detected at least two different populations in the galaxy, the age of the youngest one being around 4-6 Myr. But the more evolved stellar population, with age older than 100-200 Myr, fits an exponential intensity profile. A model which combines $85 \%$ of a young (6 Myr) population with $15 \%$ of an old (140-200 Myr) population can explain both the spectral energy distribution and the $\mathrm{H}$ Balmer and He I absorption lines observed in our spectrum. Furthermore, the NIR colors suggest that even an older stellar population, with age upper than 1-2 Gyr, is also present in IRAS $08339+6517$. Its companion galaxy is practically dominated by an old population with age $>250 \mathrm{Myr}$, although it also hosts a recent $\sim 6$ Myr starburst in its external areas. The Keplerian, ionized and cold dust masses derived for the main galaxy seem to be the usual for young starbursts.

Our spectra seem to show weak Wolf-Rayet features in IRAS $08339+6517$, but they are located only in the central knot of the galaxy. However, deeper spectra are needed to confirm the WR nature of this galaxy. We discuss that aperture effects and localization of the bursts with WR stars could play a fundamental role in the detection of this sort of massive stars in starburst galaxies. 
We have derived the SFR of IRAS 08339+6517 using multi-wavelength correlations. The $\mathrm{H} \alpha$, FIR and radio luminosities give similar values, around $9.5 M_{\odot} \mathrm{yr}^{-1}$, suggesting that the contribution of reddening by dust has been properly estimated. The high $S F R$ derived using X-ray luminosities implies massive stars formation in the last few Myr, as seems to be supported by the high rate of supernova explosions and the probable detection of a large number WR stars.

Knot B, located in the NW arm of the main galaxy, has the higher metallicity and possesses peculiar kinematics. It could be a TDG candidate because its metallicity is higher than that expected for a dwarf galaxy with its $B$-luminosity and because the kinematics of the ionized gas are decoupled from the general kinematic pattern. However, it could also be a remnant of a previous early merger because of the peculiar morphology of the galaxy and the existence of the H I tidal tail. Besides it, at the present time the interaction with the external companion galaxy is much more prominent and probably the origin of the H I tidal tail.

Finally, IRAS $08339+6517$ could be classified as a luminous compact blue galaxy (LCBG) because of its color, absolute magnitude and surface brightness. There are very few local LCBGs nowadays detected but nearly half of them have optical companions, present disturbed morphologies and/or are clearly interacting (Garland et al. 2004). An example of local LCBG is Mkn 1087 (López-Sánchez et al. 2004b), that is in interaction with two nearby galaxies and shows bridges and tails that connect dwarf surrounding objects with the main body. If interactions were the responsible of the activity in LCBGs, it would indicate that they were perhaps more common at high redshifts, as the hierarchical galaxies formation models predict (i.e., Kauffmann \& White 1993; Springel et al. 2005). This fact would also support the idea that interaction with dwarf companion objects could be an important trigger mechanism of the star formation activity in local starbursts.

Acknowledgements. We thank Mónica Rodríguez for her kindly comments on the text. We are very grateful to the anonymous referee for his/her very valuable comments and discussions that have considerably improved this paper. We would like to acknowledge Anlaug Amanda Kaas and Eric Stempels for their help with NOT observations. We are indebted to Ricardo Amorin, Ruben Sánchez and, specially, to Luzma Cairós for their help deriving the surface brightness profiles of the galaxies. A.R.L.-S. thanks John Hibbard and Lourdes Verdes-Montenegro for their valuable comments about radio observations. This work has been partially funded by the Spanish Ministerio de Ciencia y Tecnología (MCyT) under project AYA2004-07466. This research has made use of the NASA/IPAC Extragalactic Database (NED) which is operated by the Jet Propulsion Laboratory, California Institute of Technology, under contract with the National Aeronautics and Space Administration.

\section{References}

Asplund, M., Grevesse, N., Sauval, A. J, Allende-Prieto, C., \& Kiselman, D. 2004, A\&A, 417, 751

Benjamin, R. A., Skillman, E. D., \& Smits, D. P. 2002, ApJ, 569, 288 Bergvall, N., \& Östlin, G. 2002, A\&A, 390, 891

Bertilli, G., Bressan, A., Chiosi, C., Fagotto, F., \& Nasi, E. 1994, A\&AS, 106, 275
Bettoni, D., Galletta, G., \& García-Burillo, S. 2003, A\&A, 405, 5 Cairós, L. M., Vílchez, J. M., González Pérez, J. N., Iglesias-Páramo, J., \& Caon, N. 2001, AJSS, 133, 321

Calzetti, D. 2001, PASP, 113, 1149

Cannon, J. M., Skillman, E. D., Kunth, D., et al. 2004, ApJ, 608, 768

Cedrés, B. 2003, Ph.D. Thesis, Universidad de la Laguna

Condon, J. J., Helou, G., Sanders, D. B., \& Soifer, B. T. 1990, ApJS, 73,359

Condon, J. J., Anderson, M. L., \& Helou, G. 1991, ApJ, 376, 95

Condon, J. J. 1992, ARA\&A, 30, 575

Condon, J. J., Huang, Z.-P., Yin, Q. F., \& Thuan, T. X. 1992, ASPC, 31,79

Condon, J. J., Cotton, W. D., \& Broderick, J. J. 2002, AJ, 124, 675

Conti, P. S. 1991, ApJ, 377, 115

Contini, T., Treyer, M. A., Sullivan, M., \& Ellis, R. S. 2002, MNRAS, 330,75

Denicoló, G., Terlevich, R., \& Terlevich, E. 2002, MNRAS, 330, 69

Dopita, M. A., Kewley, L. J., Heisler, C. A., \& Sutherland, R. S. 2000, ApJ, 542, 224

Dopita, M. A., Pereira, M., Kewley, L. J., \& Capaccioli 2002, ApJS, 143,47

Duc, P. A., Brinks, E., Springel, V., et al. 2000, AJ, 120, 1238

Erb, D. K., Shapley, A. E., Steidel, C. C., et al. 2003, ApJ, 591, 101

Fernandes, I. F., de Carvalho, R., Contini, T., \& Gal, R. R. 2004, MNRAS, 355, 728

Fioc, M., \& Rocca-Volmerange, B. 1997, A\&A, 326, 950

Gallagher, J. S. III, Hunter, D. A., \& Tutukov, A. V. 1984, ApJ, 284, 544

Garland, C. A., Pisano, D. J., Williams, J. P., Guzmán, R., \& Castander, F. J. 2004, ApJ, 615, 689

Garnett, D. R. 1992, AJ, 103, 1330

Garnett, D. R., Shields, G. A., Peimbert, M., et al. 1999, ApJ, 513, 168

Gioia, I. M., Maccacaro, T., Schild, R. E., et al. 1990, ApJS, 72, 567

González-Delgado, R. M., Leitherer, C., Heckman, T., et al. 1998, ApJ, 495, 698

González-Delgado, R. M., \& Leitherer, C. 1999, ApJS, 125, 479

González-Delgado, R. M., Leitherer, C., \& Heckman, T. 1999, ApJS, 125,489

Grebel, E. K. 1999, The Stellar Content of the Local Group, ed. P. Whitelock, \& R. Cannon (ASP), IAU Symp., 192, 17

Guseva, N. G., Izotov, Y. I., Papaderos, P., et al. 2001, A\&A, 378, 756

Guzmán, R., Koo, D. C., Faber, S. M., et al. 1996, ApJ, 460L, 5

Guzmán, R., Jadren, A., Koo, D. C., et al. 1998, ApJ, 495, L13

Guzmán, R., Östlin, G., Kunth, D., et al. 2003, ApJ, 586, L45

Hammer, F., Gruel, N., Thuan, T. X., Flores, H., \& Infante, L. 2001 ApJ, 550, 570

Hamuy, M., Walker, A. R., Suntzeff, N. B., et al. 1992, PASP, 104, 533

Hibbard, J., Guhathakurta, P., van Gorkom, J. H., \& Schweizer, F. 1994, AJ, 107, 67

Hibbard, J. E., \& van Gorkom, J. H. 1996, AJ, 111, 655

Howarth, I. D., \& Murray, J. 1990, SERC Starlink User Note No. 50

Huang, J. H., Gu, Q. S., Ji, L., et al. 1999, ApJ, 513, 215

Huchtmeier, W. K., Sage, L. J., \& Henkel, C. 1995, A\&A, 300, 675

Izotov, Y. I., \& Thuan, T. X. 1999, ApJ, 511, 639

Izotov, Y. I., \& Thuan, T. X. 2004, ApJ, 616, 768

Izotov, Y. I., Papaderos, P., Guseva, N. G., Fricke, K. J., \& Thuan, T. X. 2004, A\&A, 421, 539

Kauffmann, G., \& White, S. D. M. 1993, MNRAS, 261, 921

Kennicutt, R. C. Jr. 1984, ApJ, 287, 116

Kennicutt, R. C. Jr. 1998, ApJ, 498, 541

Kent, S. M., Dame, T. M., \& Fazio, G. 1991, ApJ, 378, 131

Kewley, L. J., Heisler, C. A., Dopita, M. A., et al. 2000, ApJ, 530, 704

Kewley, L. J., Geller, M. J., \& Jansen, R. A. 2004, AJ, 127, 2002 
Kewley, L. J., Jansen, R. A., \& Geller, M. J. 2005, PASP, 117, 227

Kniazev, A. Y., Pustilnik, S. A., Grebel, E. K., Lee., H., \& Pramsku, A. G. 2004, ApJS, 153, 429

Koo, D. C., Guzmán, R., Faber, S. M., et al. 1995, ApJ, 440, L49

Kunth, D., Mas-Hesse, J. M., Terlevich, E., et al. 1998, A\&A, 334, 11

Landolt, A. U. 1992, AJ, 104, 340

Leitherer, C. 1994, RvMA, 7, 73

Leitherer, C., Schaerer, D., Goldader, J. D., et al. 1999, ApJS, 123, 3, STARBURST99

Lípari, S., Terlevich, R., Díaz, R. J., et al. 2003, MNRAS, 340, 289

Loose, H.-H., \& Thuan, T. X. 1986, ApJ, 309, 59

López-Sánchez, Á. R., \& Esteban 2003, Rev. Mex. Astron. Astrofis. Conf. Ser., 18, 48

López-Sánchez, Á. R., Esteban, C., \& Rodríguez, M. 2004, ApJS, 153, 243

López-Sánchez, Á. R., Esteban, C., \& Rodríguez, M. 2004, A\&A, 428, 445

López-Sánchez, Á. R. 2006, Ph.D. Thesis, Universidad de la Laguna, Tenerife, Spain, in preparation

Margon, B., Anderson, S. F., Mateo, M., Fich, M., \& Massey, P. 1988, ApJ, 334, 597

Massey, P., Strobel, K., Barnes, J. V., \& Anderson, E. 1988, ApJ, 328, 315

Mas-Hesse, J. M., Kunth, D., Tenorio-Tagle, G., et al. 2003, ApJ, 598, 858

Mazzarrella, J. M., \& Boronson, T. A. 1993, ApJS, 85, 27

Méndez, D. I., \& Esteban, C. 2000, A\&A, 359, 493

Melbourne, J., \& Salzer, J. J. 2002, AJ, 123, 2302

Moshir, M., et al. 1990, in The IRAS Faint Source Catalog, Version 2.0 (Pasadena: IPAC)

Noeske, K. G., Papaderos, P., Cairós, L. M., \& Fricke, K. J. 2003, A\&A, 410, 481

Peimbert, M., \& Costero, R. 1969, Bol. Obs. Tonantzintla y Tacubaya, 5,3

Phillips, A. C., Guzmán, R., Gallego, J., et al. 1997, ApJ, 489, 543

Pilyugin, L. S. 2001, A\&A, 369, 594
Pindao, M., Schaerer, D., González-Delgado, R. M., \& Stasińska, G. 2002, A\&A, 394, 443

Popescu, C. C., \& Hopp, U. 2000, A\&AS, 142, 247

Pustilnik, S., Kniazev, A., Pramskij, A., et al. 2004, A\&A, 419, 469

Quinet, P. 1996, A\&AS, 116, 573

Ranalli, P., Comastri, A., \& Setti, G. 2003, A\&A, 399, 39

Richer, M. G., \& McCall, M. L. 1995, ApJ, 445, 642

Rieke, G. H., \& Lebofsky, M. J. 1985, ApJ, 288, 618

Rodríguez, M., \& Rubin, R. H. 2005, ApJ, 626, 900

Roussel, H., Sauvage, M., Vigroux, L., \& Bosma, A. 2001, A\&A, 372, 427

Schaerer, D., \& Vacca, W. C. 1998, ApJ, 497, 618

Schaerer, D., Contini, T., \& Pindao, M. 1999, A\&AS, 136, 35

Schaerer, D., Guseva, N. G., Izotov, Yu. I., \& Thuan, T. X. 2000, A\&A, 362,53

Schlegel, D. J., Finkbeiner, D. P., \& Davis, M. 1998, ApJ, 500, 525

Seaton, M. J. 1979, MNRAS, 187, 73

Skillman, E. D., Côté, S., \& Miller, B. W. 2003, AJ, 125, 593

Smith, L. F., Shara, M. M., \& Moffat, A. F. J. 1996, MNRAS, 281, 163

Springel, V., White, S., et al. 2005, Nature, 435, 629

Stasińska, G., Schaerer, D., \& Leitherer, C. 2001, A\&A, 370, 1

Stevens, I. R., \& Strickland, D. K. 1998, MNRAS, 301, 215

Storey, P. J., \& Hummer, D. G. 1995, MNRAS, 272, 41

Tran, H. D., Sirianni, M., Ford, H. C., et al. 2003, ApJ, 585, 750

Vacca, W. D., \& Conti, P. S. 1992, ApJ, 401, 543

Véron, P., Gonçalves, A. C., \& Véron-Cetty, M.-P. 1997, A\&A, 319, 52

Villar-Martín, M., Cerviño, M., \& González-Delgado, R. M. 2004, MNRAS, 355, 1132

Weilbacher, P. M., Duc, P.-A., \& Fritze-v. Alvensleben, U. 2003, A\&A, 397, 545

Werk, J. K., Jangren, A., \& Salzer, J. J. 2004, ApJ, 617, 1004

Wiklind, T. 1989, A\&A, 219, L11

Yun, M. S., Reddy, N. A., \& Condon, J. J. 2001, ApJ, 554, 803

Zaritski, D., Kennicutt, R. C. Jr., \& Huchra, J. P. 1994, ApJ, 420, 87

Zhang, H. L. 1996, A\&AS, 119, 523 
Á. R. López-Sánchez et al.: SF and stellar populations in the LCBG IRAS 08339+6517, Online Material $p 1$

\section{Online Material}


Á. R. López-Sánchez et al.: SF and stellar populations in the LCBG IRAS 08339+6517, Online Material p 2

Table 4. Dereddened line intensity ratios with respect to $I(\mathrm{H} \beta)=100$ for the two galaxies and the selected knots of IRAS $08339+6517$. Correction for underlying stellar absorption in H I Balmer lines has been also applied.

\begin{tabular}{|c|c|c|c|c|c|c|c|}
\hline Line & $f(\lambda)$ & IRAS 08339+6517 & $\# 1$ & $\# 2$ & A & $\mathrm{B}$ & Companion \\
\hline $3727[\mathrm{O} \mathrm{III}]^{a}$ & 0.27 & $288 \pm 11$ & $222 \pm 20$ & $242 \pm 17$ & $322 \pm 115$ & $197 \pm 100$ & $310 \pm 44$ \\
\hline 3869 [Ne III] & 0.23 & $16.8 \pm 3.2$ & $16.7 \pm 4.9$ & $11.5 \pm 2.4$ & $\ldots$ & $\ldots$ & 17.6: \\
\hline $3889 \mathrm{He} \mathrm{I}+\mathrm{H} 8$ & 0.22 & $10.6 \pm 3.1$ & 5.3: & 8.5: & $\ldots$ & $\ldots$ & $\ldots$ \\
\hline $3968[\mathrm{Ne}$ III $]+\mathrm{H} 7$ & 0.21 & $9.7 \pm 0.9$ & 5.8: & 4.3: & $\ldots$ & $\ldots$ & 6.9: \\
\hline $4101 \mathrm{H} \delta$ & 0.18 & $25.9 \pm 3.1$ & $25.9 \pm 3.6$ & $25.9 \pm 4.6$ & $\ldots$ & $\ldots$ & 23: \\
\hline $4144 \mathrm{He} \mathrm{I}$ & 0.17 & 2.4: & $\ldots$ & $\ldots$ & $\ldots$ & $\ldots$ & $\ldots$ \\
\hline $4340 \mathrm{H} \gamma$ & 0.135 & $46.9 \pm 3.2$ & $46.9 \pm 3.7$ & $46.9 \pm 3.9$ & $\ldots$ & $\ldots$ & 42: \\
\hline 4363 [O III] & 0.13 & $0.70:$ & $0.8:$ & 1.0: & $\ldots$ & $\ldots$ & $\ldots$ \\
\hline $4471 \mathrm{He} \mathrm{I}$ & 0.10 & $3.17 \pm 0.50$ & 1.4: & 1.3: & $\ldots$ & $\ldots$ & $\ldots$ \\
\hline 4658 [Fe III $]$ & 0.05 & $1.20 \pm 0.55$ & 0.8: & $\ldots$ & $\ldots$ & $\ldots$ & $\ldots$ \\
\hline $4686 \mathrm{He}$ II & 0.05 & $\ldots$ & 1.9: & $\ldots$ & $\ldots$ & $\ldots$ & $\ldots$ \\
\hline $4861 \mathrm{H} \beta$ & 0.00 & $100 \pm 3$ & $100 \pm 4$ & $100 \pm 4$ & $100 \pm 30$ & $100 \pm 25$ & $100 \pm 13$ \\
\hline 4959 [O III] & -0.02 & $56 \pm 2$ & $63 \pm 4$ & $49 \pm 4$ & $34:$ & $64 \pm 24$ & $44 \pm 9$ \\
\hline 5007 [O III] & -0.03 & $168 \pm 4$ & $190 \pm 10$ & $163 \pm 9$ & $102 \pm 36$ & $211 \pm 62$ & $135 \pm 19$ \\
\hline $5200[\mathrm{~N} \mathrm{I}]$ & -0.05 & $2.38 \pm 0.16$ & 2.1: & 2.3: & $\ldots$ & $\ldots$ & $\ldots$ \\
\hline 5516 [Cl III] & -0.15 & $0.6:$ & $\ldots$ & $\ldots$ & $\ldots$ & $\ldots$ & $\ldots$ \\
\hline 5538 [Cl III] & -0.16 & $0.5:$ & $0.7:$ & $\ldots$ & $\ldots$ & $\ldots$ & $\ldots$ \\
\hline $5755[\mathrm{~N} \mathrm{II}]$ & -0.21 & $0.82 \pm 0.29$ & 1.1: & 0.6 : & $\ldots$ & $\ldots$ & $\ldots$ \\
\hline $5876 \mathrm{He} \mathrm{I}$ & -0.23 & $11.6 \pm 0.8$ & $13.3 \pm 1.8$ & $11.7 \pm 1.9$ & $\ldots$ & $\ldots$ & $11 \pm 4$ \\
\hline 6300 [O I] & -0.30 & $5.5 \pm 0.8$ & $3.7 \pm 1.1$ & $5.0 \pm 1.0$ & $\ldots$ & $\ldots$ & $\ldots$ \\
\hline 6548 [N II] & -0.34 & $29.4 \pm 1.5$ & $27.1 \pm 2.4$ & $26.9 \pm 2.9$ & $23 \pm 9$ & $26 \pm 6$ & $17.6 \pm 4.9$ \\
\hline $6563 \mathrm{H} \alpha$ & -0.34 & $286 \pm 10$ & $286 \pm 14$ & $286 \pm 15$ & $286 \pm 83$ & $286 \pm 78$ & $286 \pm 37$ \\
\hline 6584 [N II] & -0.34 & $79.3 \pm 3.1$ & $78.9 \pm 5.6$ & $76.4 \pm 5.5$ & $62 \pm 19$ & $81 \pm 19$ & $58.2 \pm 9.3$ \\
\hline $6678 \mathrm{He} \mathrm{I}$ & -0.35 & $2.91 \pm 0.38$ & 2.3: & $3.4:$ & $\ldots$ & $\ldots$ & $\ldots$ \\
\hline$F(\mathrm{H} \beta)^{b}$ & $\ldots$ & $192 \pm 6$ & $36.5 \pm 1.3$ & $28.7 \pm 1.0$ & $0.329 \pm 0.097$ & $0.324 \pm 0.081$ & $2.39 \pm 0.31$ \\
\hline$C(\mathrm{H} \beta)$ & $\ldots$ & $0.22 \pm 0.02$ & $0.30 \pm 0.02$ & $0.29 \pm 0.02$ & $0.18 \pm 0.03$ & $0.25 \pm 0.03$ & $0.18 \pm 0.03$ \\
\hline$W_{\mathrm{abs}}^{c}$ & $\ldots$ & $1.8 \pm 0.1$ & $1.1 \pm 0.1$ & $0.9 \pm 0.1$ & $1.5 \pm 0.1$ & $1.5 \pm 0.1$ & $1.5 \pm 0.1$ \\
\hline$-W(\mathrm{H} \alpha)^{c}$ & $\ldots$ & $104 \pm 2$ & $140 \pm 6$ & $93 \pm 4$ & $18 \pm 2$ & $26 \pm 3$ & $27 \pm 0.02$ \\
\hline$-W(\mathrm{H} \beta)^{c}$ & $\ldots$ & $19.0 \pm 0.8$ & $25 \pm 2$ & $17.6 \pm 1.5$ & $5.6 \pm 1.1$ & $6.7 \pm 0.7$ & $7.5 \pm 0.2$ \\
\hline$-W(\mathrm{H} \gamma)^{c}$ & $\ldots$ & $5.9 \pm 0.3$ & $4.9 \pm 0.4$ & $5.7 \pm 0.6$ & $\ldots$ & $\ldots$ & $\ldots$ \\
\hline$-W(\mathrm{H} \delta)^{c}$ & $\ldots$ & $2.1 \pm 0.4$ & $1.2 \pm 0.2$ & $2.3 \pm 0.4$ & $\ldots$ & $\ldots$ & $\ldots$ \\
\hline$-W([\mathrm{O} \mathrm{III}])^{c}$ & $\ldots$ & $27.2 \pm 0.9$ & $24 \pm 1$ & $29 \pm 1$ & $4.0 \pm 0.4$ & $6.7 \pm 0.4$ & $7.9 \pm 0.4$ \\
\hline
\end{tabular}

${ }^{a}$ [O II] $\lambda \lambda 3726,3729$ emission flux. ${ }^{b}$ In units of $10^{-15} \mathrm{erg} \mathrm{s}^{-1} \mathrm{~cm}^{-2} \cdot{ }^{c}$ In units of $\AA$. 